\title{
Particle simulations of divergent and convergent radial electron flows in cylindrical Pierce diodes
}

\author{
M. Virgínia Alves \\ Instituto Nacional de Pesquisas Espaciais, P.O. Box 515, 12201-970, São José dos Campos, SP, Brazil \\ Fausto T. Gratton, Graciela Gnavi, and Cesar H. Moreno \\ Instituto de Física del Plasma, CONICET, Departamento de Física, Facultad de Ciencias \\ Exactas y Naturales, Universidad de Buenos Aires, Ciudad Universitaria, Pabellón 1, 1428 Buenos Aires, \\ Argentina
}

(Received 21 February 1997; accepted 17 April 1997)

\begin{abstract}
A special class of Pierce diodes, that consists of a pair of grounded coaxial cylindrical electrodes, with electrons streaming radially between them, is studied. The full set of roots of the dispersion relation of the Pierce instability is obtained by solving the dispersion relation numerically. The nonlinear behaviour of the system is then investigated using particle simulation. It is shown that the behaviour of cylindrical systems depends on two nondimensional parameters. Linear and nonlinear properties are different for convergent and divergent configurations. Several remarkable differences between cylindrical and planar Pierce diodes are reported. (C) 1997 American Institute of Physics. [S1070-664X(97)04807-6]
\end{abstract}

\section{INTRODUCTION}

The instability and associated limiting current for a neutralized electron beam drifting between planar grounded grids (Pierce's diode) has been extensively studied in experiments, theory (see Ref. 1 and references therein), and particle simulations. ${ }^{2,3}$ Pierce-like configurations appear in a variety of plasma systems of current interest, including inertial fusion, collective process accelerators, Q-machines, and high power microwave amplification.

The fact that in a bounded plasma, contrary to what happens in an infinite system, a monoenergetic electron beam alone may become unstable when drifting freely between grounded electrodes (excluding any interaction with ions) is unexpected. It has attracted the attention of researchers over many years. A survey ${ }^{1}$ of this topic (dated 1987) covers about 150 references. By far, the existing literature is devoted to planar diodes.

For a long time (after Pierce's 1944 paper $^{4}$ ) the instability properties were known near the threshold only. Assuming linear modes varying as $\exp (\gamma t)$, the roots of the dispersion relation were examined near $\gamma=0$. Later it was pointed out by Pierce himself, ${ }^{5}$ that the unstable behaviour is related to the existence of bifurcations, i.e., the coexistence of two equilibrium states of the beam, one being the unperturbed beam state (with constant speed and uniform density) and the other a new steady state with spatial variations of density and velocity of the beam. The theoretical condition for the existence of marginal states, $\gamma=0$ (assuming neutrality and fixed ions) is $\alpha=(2 n+1) \pi$, for $n=0,1,2, \ldots$, where $\alpha$, the Pierce diode parameter, is given by $\alpha=\omega_{p} L / v_{b}\left(\omega_{p}\right.$ is the plasma frequency computed with the beam density; $L$ is the interelectrode distance; $v_{b}$ is the free streaming beam speed). The threshold for the first unstable mode corresponds to $n=0$. For $\alpha$ greater, but close to $\pi$, the instability was found to be monotonic in time. The rather complex dispersion relation was investigated numerically afterwards. ${ }^{6}$ As a function of increasing $\alpha$, a rich sequence of modes was found. Mono- tonically growing and damped modes alternate, first with oscillatory growing solutions (overstability), then with oscillatory damped modes. Segments of $\alpha$ values with different properties were discerned. Monotonically unstable and oscillatory growing modes are separated by narrow $\alpha$ intervals, where only damped and oscillatory damped modes exists. We shall call these $\alpha$ intervals, stability islands. The full set of roots of the dispersion relation was then examined in Ref. 7 , where the linear theory of the initial value problem for the Pierce diode was studied in detail. Several other aspects, such as the coupling of the diode with external circuits, were also studied (see, e.g., literature quoted in Ref. 1). The set of roots of the linear dispersion relation, for grounded electrodes, was again considered in Refs. 8 and 9, in connection with studies of nonlinear coupling of modes, bifurcation of solutions, and nonlinear steady states. The possibility of deterministic chaos arising in Pierce diodes was revealed in these works. At the same time, numerical simulations, ${ }^{2,8,10,11}$ began to provide a comprehensive understanding of the nonlinear behaviour of the system, and contributed to the coming of age of the subject. Recent literature indicates a persistent interest in Pierce diodes, and shows new features (Ref. 12 and papers quoted therein) like the subtle effects produced by a spread in velocity of the electron beam.

The theory of a different type of Pierce diode was recently given in Ref. 13 where, departing from the planar setting, electrons drifting radially in the space between two coaxial cylindrical grids, both grounded, are considered. In the unperturbed steady state the electrons flow radially with a constant average velocity $v_{r}= \pm v_{b}$. The flow is convergent when the electrons move from the outer cylinder (emitter) to the inner cylinder (collector); when the motion is in the opposite direction, from inner to outer electrode, the flow is divergent. Ions are present to ensure charge neutrality, but in the model the dynamics of the ions is ignored. Thus, without additional changes of the theory, ions may also be assumed to flow together with the electrons, describing a current free system if so is desired. For geometrical reasons, both elec- 
trons and ions have an unperturbed density distribution $n=A / r$ in the interelectrode space. This configuration must be distinguished from the cylindrical waveguide device (also studied in the context of Pierce diodes, e.g., Ref. 14) where the beam moves along the axis, and which is akin to a planar diode with bounded cross section.

Electronic devices with cylindrical and spherical electrodes were in fact called Pierce electrodes (for a description see, for instance, Ref. 15). For a technological review of electrodes with different shapes, see Ref. 16. Experiments with electron flows in cylindrical configurations are reported in Ref. 17. Fusion studies have also considered convergent flows of ions and electrons (see, for example, Refs. 18 and 19, for spherical geometry).

As shown in Ref. 13, a flow directed from an inner toward an outer cylindrical electrode (divergent flow) is interesting for microwave signal amplification. Stability of convergent flows (from outer to inner grids) is relevant for inertial confinement schemes that focus a neutralized ion beam on a target. ${ }^{20}$ The co-moving electrons are used to provide charge and current neutralization. At a much smaller beam energy the same configuration is interesting also for ion implantation, or plasma coating experiments.

Compared with the research done on planar Pierce diodes, much less is known about cylindrical Pierce configurations. The dispersion relation of linear modes for the cylindrical system was derived in Ref. 13 [see Eq. (16)]. However, because of its considerable complexity due to terms containing single and double indefinite integrals of products of Bessel functions and exponentials, it cannot be solved analytically in general, while the numerical search for roots is not a simple task. Thus Pierce instability was studied in Ref. 13 only near the marginal states [where $\gamma=0$; see Eq. (17)].

We have recalled some important facts about planar Pierce diodes, and outlined the research status on cylindrical Pierce diodes. The aim of the present paper is to contribute to the understanding of cylindrical systems in two basic aspects, not examined before. First, we analyze the full set of roots of the dispersion relation of the Pierce instability by solving numerically the dispersion relation. Then, we study systematically (for the first time, as far as we know) the nonlinear behaviour by particle simulation.

While planar diodes depend on a single nondimensional parameter, the behaviour of cylindrical systems depends on two parameters. In addition, linear and nonlinear properties are different for convergent and divergent configurations. Therefore, the description of the results is rather extended. We also give some attention to the role played in the evolution of the instability by steady state nonlinear solutions that bifurcate at the critical $\gamma=0$ values. Exploring these aspects, we report several remarkable differences between cylindrical and planar Pierce diodes.

The layout of the paper is as follows. In Sec. II we discuss the system configuration, the equations, and the simulation code. Specifically, in Sec. II A the equations of the model are given, and their dependence on two nondimensional numbers is shown. A brief reminder of the linear theory is given in Sec. II B, and the nonlinear code is dis-
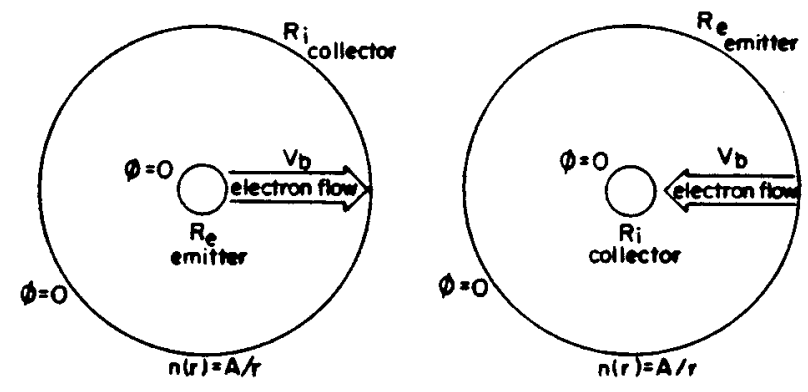

FIG. 1. Setup and geometry of the systems modeled in this paper.

cussed in Sec. II C. A full set of roots of the linear dispersion relation, computed numerically, is presented in Sec. III for three values of the ratio $x_{i}=R_{i} / R_{e}$, of the collector radius $\left(R_{i}\right)$ over the emitter radius $\left(R_{e}\right)$. Section IV gives information about bifurcations of nonlinear steady states, and preliminary indications of their stability. In Sec. V we examine the nonlinear dynamics of a pseudoplanar system $\left(x_{i} \simeq 1.3\right)$ recovering the properties of planar Pierce diodes, as a benchmark for the numerical code. In Sec. VI we show particle simulations of a highly divergent flow $\left(x_{i}=100\right)$. The nonlinear behaviour of systems focussed toward the axis is considered in Sec. VII $\left(x_{i}=0.1\right)$. Finally we give a general discussion of the results and conclusions in Sec. VIII.

\section{THEORY AND NUMERICAL CODE}

\section{A. Configuration and equations of the model}

Figure 1 shows the setup and geometry of the systems modeled here. The electrodes are coaxial cylinders. Associated with the emitter, an acceleration device (not shown) produces the electron flow. We may assume that a potential difference is applied across additional cylindrical electrodes to obtain a radial electron speed such that $e V=m v_{b}^{2} / 2$. For charge neutrality ions may be provided by the residual gas in the drifting chamber. When the electrons are comoving with injected ions, to form a charge and current neutralized flow, a more elaborate apparatus must be envisaged. In any case, before the entrance, there is an acceleration stage which is not further specified and does not take part in our consideration. After that stage, the emitter electrode set at $r=R_{e}$ is simply a grounded grid that allows the electrons to enter the free drift inter-electrode space. Similarly, the collector set at $r=R_{i}$ is a grounded grid that allows the radial flow to exit the system, toward an absorbing dump, and keeps the potential difference between electrodes equal to zero. In some practical applications the collector may represent a grounded target, but note that we do not take into account here particles reflected back into the system after hitting the target. Reflection, however, may occur inside the drifting space produced by collective effects, such as the formations of virtual cathodes. That kind of reflection is, of course, included in the code. The figure shows two basic configurations studied, with incoming (i.e., toward the axis) or outgoing radial motion, hence forming convergent or divergent bundles of electron trajectories. We assume here that the trajectories are 
well focussed through the central axis, so that the angular momentum (or impact parameter) of the particles is zero. The figure also reminds us that at the start, and leaving aside initial perturbations, the characteristic features of the flow are: constant radial speed, $v_{r}= \pm v_{b}$, and $n=A / r$, equal for both species. Therefore, no electric field exists in the interelectrode space. Under experimental conditions, the $1 / r$ density distribution is easier to achieve with comoving electronion injection. This case also has the advantage of completely avoiding a possible two stream, electron-ion instability, related to a differential speed between species. However, in view of their large mass, we ignore ion perturbations in the simulation (ions are kept fixed, with $A / r$ density) to give perspicuity to the electron dynamics, and to facilitate comparisons with the theory. ${ }^{13}$ Thus, the results we report are not sensitive to a particular ion setup, i.e., comoving with electrons, or simply as fixed neutralizing background.

The equations of the model, setting $v=v_{r}$, $E_{r}=-\partial \phi / \partial r$, and assuming a monoenergetic beam, are

$$
\begin{aligned}
& \frac{\partial v}{\partial t}+v \frac{\partial v}{\partial r}=\frac{e}{m} \frac{\partial \phi}{\partial r}, \\
& \frac{\partial(r n)}{\partial t}+\frac{\partial(r n v)}{\partial r}=0, \\
& \frac{\partial}{\partial r}\left(r \frac{\partial \phi}{\partial r}\right)=-\frac{e}{\epsilon_{0}}\left(n_{e} R_{e}-n r\right),
\end{aligned}
$$

with boundary conditions $\phi\left(R_{e}\right)=\phi\left(R_{i}\right)=0$. The beam enters at $r=R_{e}$ (density $n_{e}$, speed $v_{b}$ ) and exits from the system at $r=R_{i}$.

It is important to realize that the model depends on two nondimensional parameters only. This can be seen using the following set of nondimensional variables: $u=v / v_{b}$, $x=r / R_{e}, \quad \nu=n / n_{e}, \quad T=t / \tau$ (where $\left.\quad \tau=\left|x_{i}-1\right| R_{e} / v_{b}\right)$, $\varphi=2 e \phi / m v_{b}{ }^{2}$. In numerical experiments we use $T$, the time measured in units of the transit time $\tau$. The spatial variable $x$ is usually $r$ in units of $R_{e}$, the emitter radius. Eventually, for some comparisons, we may wish to measure $r$ in units of the interelectrode distance $\left|x_{i}-1\right| R_{e}$. Nondimensional growth rates $\operatorname{Re}(s)$ derived from theory, are given in units of $R_{e} / v_{b}$ as in Ref. 13 (divide by $\left|x_{i}-1\right|$ if transit time units are preferred).

Equations (1)-(3) are then written as follows:

$$
\begin{aligned}
& \frac{\partial u}{\partial T}+\frac{\left|x_{i}-1\right|}{2} \frac{\partial\left(u^{2}-\varphi\right)}{\partial x}=0, \\
& \frac{\partial(x \nu)}{\partial T}+\left|x_{i}-1\right| \frac{\partial(x \nu u)}{\partial x}=0, \\
& \frac{\partial}{\partial x}\left(x \frac{\partial \varphi}{\partial x}\right)=-\frac{D^{2}}{2}(1-\nu x) .
\end{aligned}
$$

Two basic numbers appear in (4)-(6), $x_{i}$ and $D=2 R_{e} \omega_{p} / v_{b}$. We define $\omega_{p}^{2}=e^{2} n_{e} / \epsilon_{0} m$ using $n_{e}$, the density at the emitter. Sometimes, for convenience we use $\alpha$, the Pierce number, defined as $\alpha=\left|x_{i}-1\right| D / 2=\tau \omega_{p}$, for cylindrical systems.

\section{B. Summary of the linear theory}

In Ref. 13 it is shown that linear modes that represent perturbations of constant velocity steady states are ruled by the following equations for the nondimensional variable $\mathscr{E} \equiv r \epsilon_{0} E_{r} / e n_{e} R_{e}^{2}$ :

$$
\begin{aligned}
& \mathscr{E}(x, \tau) \equiv \mathscr{E}(x) e^{s \tau}, \\
& \left(s-\frac{d}{d x}\right)^{2} \mathscr{E}+\frac{\mu^{2}}{x} \mathscr{E}=s \mathscr{F}_{0},
\end{aligned}
$$

where $2 \mu=D$, and $\mathscr{F}_{0}$ is a constant to be determined (together with two additional integration constants) by the boundary conditions.

If a solution for $\mathscr{E}(x)$ is known, the velocity and density perturbations are obtained from

$$
\begin{aligned}
& \mathscr{T}(x)=\left(s-\frac{d}{d x}\right) \mathscr{E}-\mathscr{F}_{0}, \\
& \mathscr{N}(x)=-\frac{d \mathscr{E}}{d x},
\end{aligned}
$$

where $\mathscr{V} \equiv \delta v / v_{b}, \mathscr{N} \equiv r \delta n / R_{e} n_{e}$.

The solution of the homogeneous equation, $\mathscr{E}_{h}$, may be expressed in terms of Bessel and Neumann functions as

$$
\mathscr{C}_{h}(x)=e^{s x} \sqrt{x}\left[c_{1} J_{1}(2 \mu \sqrt{x})+c_{2} Y_{1}(2 \mu \sqrt{x})\right],
$$

where $c_{1}, c_{2}$ are constants. From the solutions of the homogeneous equation a particular solution $\mathscr{E}_{p}$ of the inhomogeneous equation can be expressed as

$$
\begin{aligned}
\mathscr{E}_{p}(x)= & \pi s \cdot \mathscr{F}_{0} e^{s x} \sqrt{x}\left\{Y_{1}(2 \mu \sqrt{x}) \int_{1}^{x} e^{-s u} \sqrt{u} J_{1}(2 \mu \sqrt{u}) d u\right. \\
& \left.-J_{1}(2 \mu \sqrt{x}) \int_{1}^{x} e^{-s u} \sqrt{u} Y_{1}(2 \mu \sqrt{u}) d u\right\}
\end{aligned}
$$

so that, $\mathscr{E}(x)=\mathscr{E}_{h}+\mathscr{E}_{p}$.

The boundary conditions for the Pierce problem are

$$
\begin{aligned}
& \mathscr{T}(1)=0, \\
& \mathscr{N}(1)=0, \\
& \int_{x_{i}}^{1} \frac{\mathscr{E}(x)}{x} d x=0 .
\end{aligned}
$$

Equations (13) and (14) describe the injection of the unperturbed electron flow at the emitter. The last equation ensures that emitter and collector are at the same potential. This set of boundary equations forms an homogeneous linear algebraic system for $c_{1}, c_{2}$ and $\mathscr{F}_{0}$. A nontrivial solution exists when the determinant of this system is zero.

Carrying through this calculation, one obtains the dispersion equation for $s$ [equation (66) of Ref. 13] as

$$
J_{0}(2 \mu) I_{2}-Y_{0}(2 \mu) I_{1}+s e^{s} I_{0}=0,
$$

with 


$$
\begin{aligned}
I_{0}= & \int_{x_{i} \sqrt{x}}^{1} \frac{e^{s x}}{\sqrt{x}} J_{1}(2 \mu \sqrt{x}) \int_{1}^{x} e^{-s u} Y_{0}(2 \mu \sqrt{u}) d u \\
& -\int_{x_{i} \sqrt{x}}^{1} \frac{e^{s x}}{\sqrt{x}} Y_{1}(2 \mu \sqrt{x}) \int_{1}^{x} e^{-s u} J_{0}(2 \mu \sqrt{u}) d u, \\
I_{1}= & \int_{x_{i} \sqrt{x}}^{1} \frac{e^{s x}}{\sqrt{x}} J_{1}(2 \mu \sqrt{x}) d x, \\
I_{2}= & \int_{x_{i}}^{1} \frac{e^{s x}}{\sqrt{x}} Y_{1}(2 \mu \sqrt{x}) d x .
\end{aligned}
$$

Steady state solutions are determined by taking $s=0$ in (16). After integrations with $s=0$ and some algebra we find

$$
J_{0}(D) Y_{0}\left(D \sqrt{x_{i}}\right)-J_{0}\left(D \sqrt{x_{i}}\right) Y_{0}(D)=0 .
$$

Hence, there exist an infinite number of pairs $\left(x_{i}, D=2 \mu\right)$ such that (17) is satisfied. ${ }^{21}$ For these values, there are steady state solutions with nonuniform velocity. This condition gives, at the same time, the instability thresholds (equation 67 of Ref. 13).

Thus linear instability theory also depends on the two basic nondimensional parameters, $x_{i}$ and $D$ (or, alternatively, $\alpha$ ) defined above. For a given speed $v_{b}$ and emitter radius $R_{e}$, there are infinite pairs of values $R_{i}, n_{e}$ such that the system admits velocity modulated steady states. This equation plays the same role as the Pierce condition $\alpha=(2 n+1) \pi$ for planar diodes. In fact, it can be shown that planar results can be recovered in the limits $x_{i} \approx 1$ and $D \gg 1$.

Classification and understanding of linear and nonlinear behaviour, as we shall see, can be achieved, for a given $x_{i}$, through the set of threshold values $D_{1}<D_{2}<D_{3}<\cdots$ for which the eigenfrequency $s$ is zero. We shall denote the intervals $0<D<D 1, D 1<D<D 2, D 2<D<D 3, \cdots$ as regions $1,2,3, \ldots$ of the parameter space, respectively. For example, given $x_{i}=100$, we find $D_{1}=0.3314$, $D_{2}=0.6858, \ldots$. The system is linearly stable below the first threshold, i.e., for $D<D_{1}$, corresponding to region 1 .

\section{The nonlinear code}

Particle simulation methods have been well established as an effective technique for studying the nonlinear properties of plasma. The simulations presented here were done using the particle-in-cell (PIC) code PDC1 (Plasma Device Cylindrical one-dimensional $)^{22-24}$ in a workstation version XPDC1. This PIC code is one-dimensional, radial, electrostatic, and simulates a plasma contained between two concentric cylinders that can be coupled to an external RLC (resistive, inductive, and/or capacitive) circuit and/or $\mathrm{rf}$ source, i.e., it is not specialized to the cylindrical Pierce diode with radial flow. The original code has been modified in order to have an initial density that varies as $n_{0} \propto 1 / r$. During the simulations the imposed boundary conditions ensure that electrons are injected at a constant rate from the cathode (inner electrode for a divergent beam, and outer for a con- vergent one). Once the electrons reach the collector they are absorbed, contributing to the external surface charge. ${ }^{25}$ Both electrodes are kept at the same potential.

Fields are calculated on a grid of 200 points using a finite-difference Poisson scheme (equivalent to the flux conserving method ${ }^{26}$ ). Poisson's equation is solved in each time step with the given boundary conditions. The code has been described in detail in Refs. 22, 23, 25. All runs were initialized by prescribing electron spatial distributions which differ only slightly from the linear equilibrium $\left(n_{b}(r) \propto 1 / r\right.$, $\left.v_{b}(r)=v_{b}\right)$. A background of immobile ions with the same density profile was assumed. For reference, all runs discussed in this paper were standardized to involve some 30000 simulation particles (the precise number varies during each run). The input parameters have been determined from a given value of $D$ that specifies the region of interest for a particular value of $x_{i}$. The time step is determined ensuring that the Courant-Friedrichs-Levy (CFL) condition, $\gamma_{c}=v_{\max } \Delta t / \Delta x<1$, is satisfied. ${ }^{26} \mathrm{~A}$ value $v_{\max }=3 v_{b}$ is assumed, after concluding from a series of numerical experiments that this is an upper bound for the velocity. This condition means that no disturbance can propagate more than $\Delta x$ in a time step $\Delta t$, and avoids numerical instability.

\section{SOLUTIONS OF THE LINEAR DISPERSION RELATION}

The roots of (16) are obtained numerically, following several branches of the numerical solution. Starting from a critical pair $\left(x_{i}, D\right)$ corresponding to $s=0$ and fixing $x_{i}, D$ is advanced by steps identifying intervals where the left-hand side (LHS) of (16) changes sign. Then a real root $s$ is determined by bisection, and the procedure is repeated along a particular branch. Regarding complex roots, it is observed that they appear by coalescence of two real roots, for a $D$ value rather close to the critical one. Given a starting seed for $\left(x_{i}, D\right)$, the complex plane $s$ is searched with variable size steps, both in real and imaginary components, looking for zeros of (16) (standard solver procedures are used, like the gradient method and Romberg extrapolations). Efficient evaluation of double and simple integrals, and quality of prediction of an approximation of the root for the next $D$ step, are important considerations to alleviate the time-consuming task of plotting solutions of (16).

We have examined the cases $x_{i}=3.1765$, which is not far from a planar diode and, as a benchmark for the particle code, $x_{i}=1.3049$, which is closer to it. The numerical experiments where extended up to region 6 . Since we want to give attention to those cases that depart most from the planar, i.e., cylindrical diodes with $x_{i} \gg 1$ and $x_{i} \ll 1$, where curvature plays an important role, a series of numerical experiments have been performed with $x_{i}=100$ and $x_{i}=0.1$. We now present the full set of roots of the linear dispersion relation (16), for the values $x_{i}=0.1, x_{i}=3.1765$, and $x_{i}=100$.

Figure 2(a) corresponds to $x_{i}=0.1$, a convergent diode, and gives $\operatorname{Re}(s)$ as a function of $D$. Six nonoscillatory modes are shown with full lines, corresponding to damped or monotonically growing perturbations. For $D<4.5$, region 1 , the system is stable. Region 2 corresponds to the interval $4.5<D<9.2$. In this region the diode is unstable. A second 

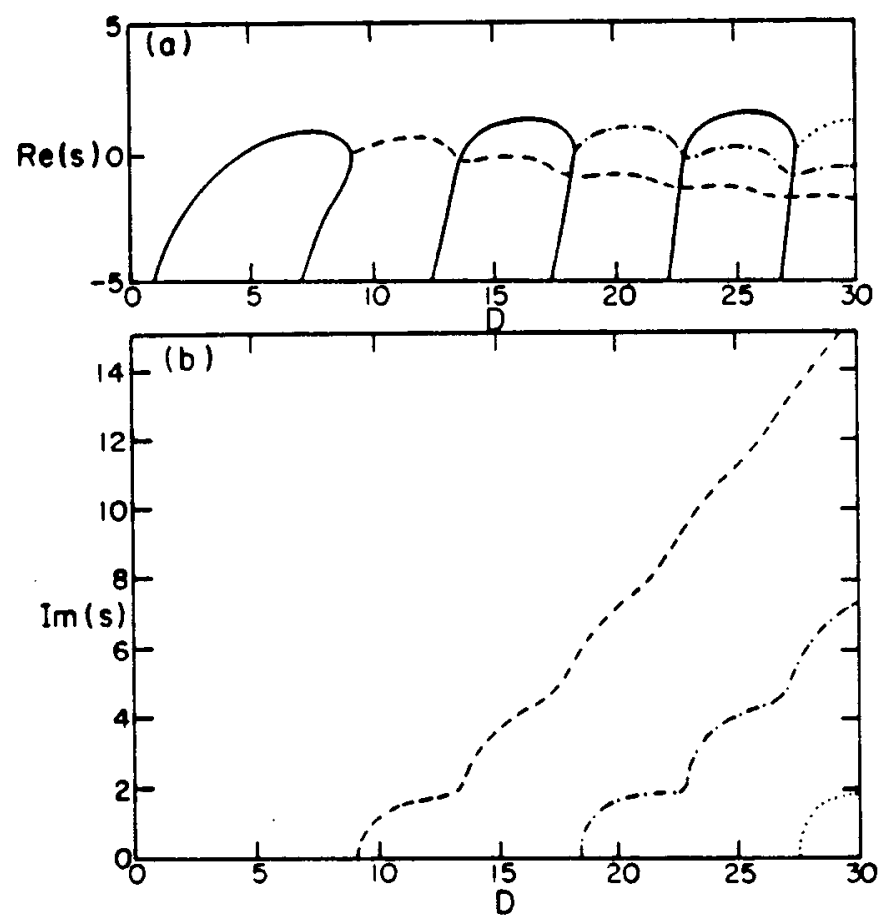

FIG. 2. Roots of the dispersion relation for $x_{i}=0.1$. (a) $\operatorname{Re}(s)$ as a function of $D ;($ b) $\operatorname{Im}(s)$ as a function of $D$.

damped mode that tends to merge with the unstable mode near the end of the interval can also be seen here. However, it must be pointed out that for a fixed value of $D>0$, an infinite succession of increasingly damped modes exists. The extensions of the five slanted lines after the first mode can be found in regions 1 and 2 , if we look at sufficiently large negative values of $\operatorname{Re}(s)$. The complete succession of modes for a diode with fixed $\left(x_{i}, D\right)$, is needed to represent, by superposition, the evolution of an arbitrary initial perturbation.

Above the limit of region 2, the damped mode of region 2 becomes a growing one. Very close to this limit, in region 3 , the two real positive roots coalesce and two complex conjugate roots appear as $D$ increases. The real and imaginary parts of the overstable mode are shown with dashed lines in Figs. 2(a) and 2(b), respectively. The oscillatory growing mode becomes oscillatory damped at $D \simeq 13.4$, where the ondulating dashed line sinks slowly toward negative $\operatorname{Re}(s)$ values. The oscillatory damped mode coexists with the third nonoscillatory mode that becomes unstable at $D \simeq 13.8$. For $13.4<D<13.8$ both modes have $\operatorname{Re}(s)<0$ and are stable. Thus, a small stability island is formed near the end of region 3 , limited by the beginning of region 4 . In region 4 the first oscillatory mode rises again to values of $\operatorname{Re}(s)>0$, within the range $15.3<D<16.0$, where it shows a weak instability with very small growth rates. The flow is in any case unstable in region 4 due to the third nonoscillatory mode.

The second oscillatory mode is represented in Figs. 2(a) and 2(b) by a dashed and dotted line and shows a general behaviour similar to the first one. It is generated again by coalescence of two real roots, near 18.4, the beginning of region 5 . The second oscillatory mode is overstable in most
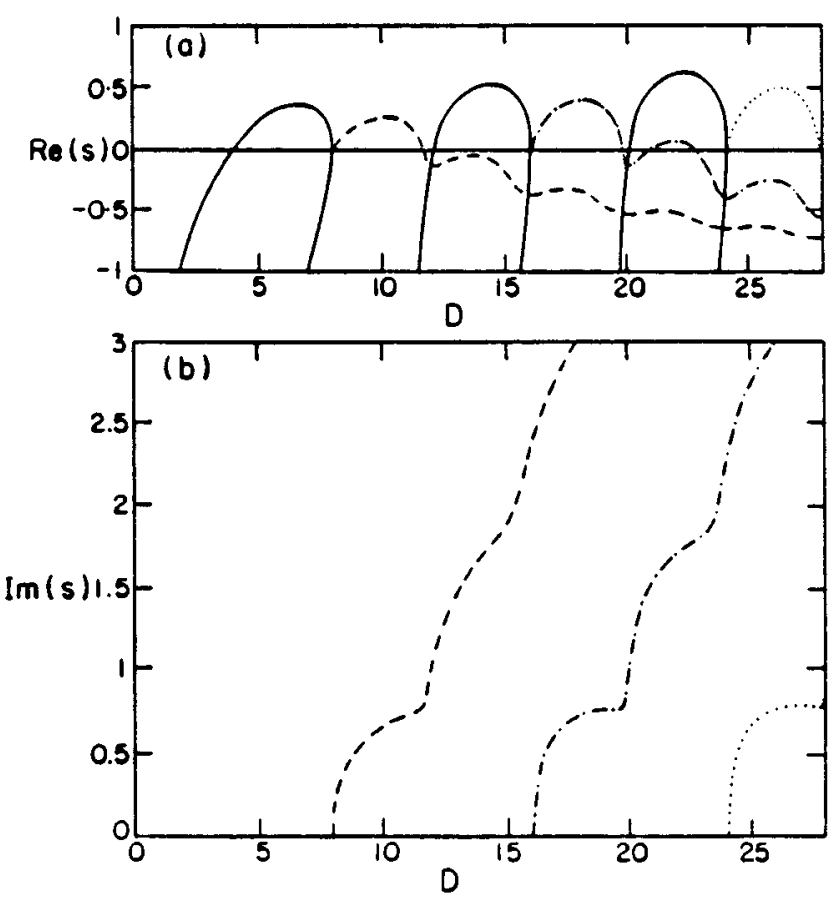

FIG. 3. Roots of the dispersion relation for $x_{i}=3.1765$. (a) $\operatorname{Re}(s)$ as a function of $D$; (b) $\operatorname{Im}(s)$ as a function of $D$.

of region 5, except near the end, where a second stability island is apparent around $22.8<D<23$, in connection with the fifth monotonic mode, which is also stable there. Afterwards, the second oscillatory mode becomes overstable again in the range $23.7<D<26.4$ inside region 6 , and then stabilized at last, it sinks with ondulations in the $\operatorname{Re}(s)<0$ halfplane. In the same figure, we can see the beginning of the third oscillatory mode (dotted line). Region 6 shows two oscillatory modes and a monotonic unstable mode, while region 7 has an overstable mode and two oscillatory damped modes (besides the infinite set of monotonic damped modes, present in all regions). From Fig. 2(b) it is apparent that $\operatorname{Im}(s)$, the frequency of the oscillatory modes, increases as $D$ increases.

The roots of the dispersion relation for the case $x_{i}=3.1765$, which correspond to a divergent flow, are plotted in Figs. 3(a) and 3(b), and show features similar to the previous case. We may note, however, that the first oscillatory mode (dotted line) shows only one unstable range, $8<D<11.5$, contained in region 3 and remains stable with increasing $D$. A stability island is formed after the first oscillatory mode becomes damped and up to the start of the third monotonically unstable mode, $11.5<D<12$. The second oscillatory mode (dashed-dotted line) is overstable in region 5, except for a second stability island in the interval $19.9<D<20$. It has another overstable range for $20.9<D<22.9$ and becomes oscillatory damped for $D>22.9$. The nondimensional growth rates are roughly half of those of the precedent case.

Finally, in Figs. 4(a) and 4(b), we give $\operatorname{Re}(s)$ and $\operatorname{Im}(s)$ for the highly divergent case $x_{i}=100$. Figure 4(c) shows a magnified particular: the merging of two real roots to produce the first overstable mode. This effect is similar to 

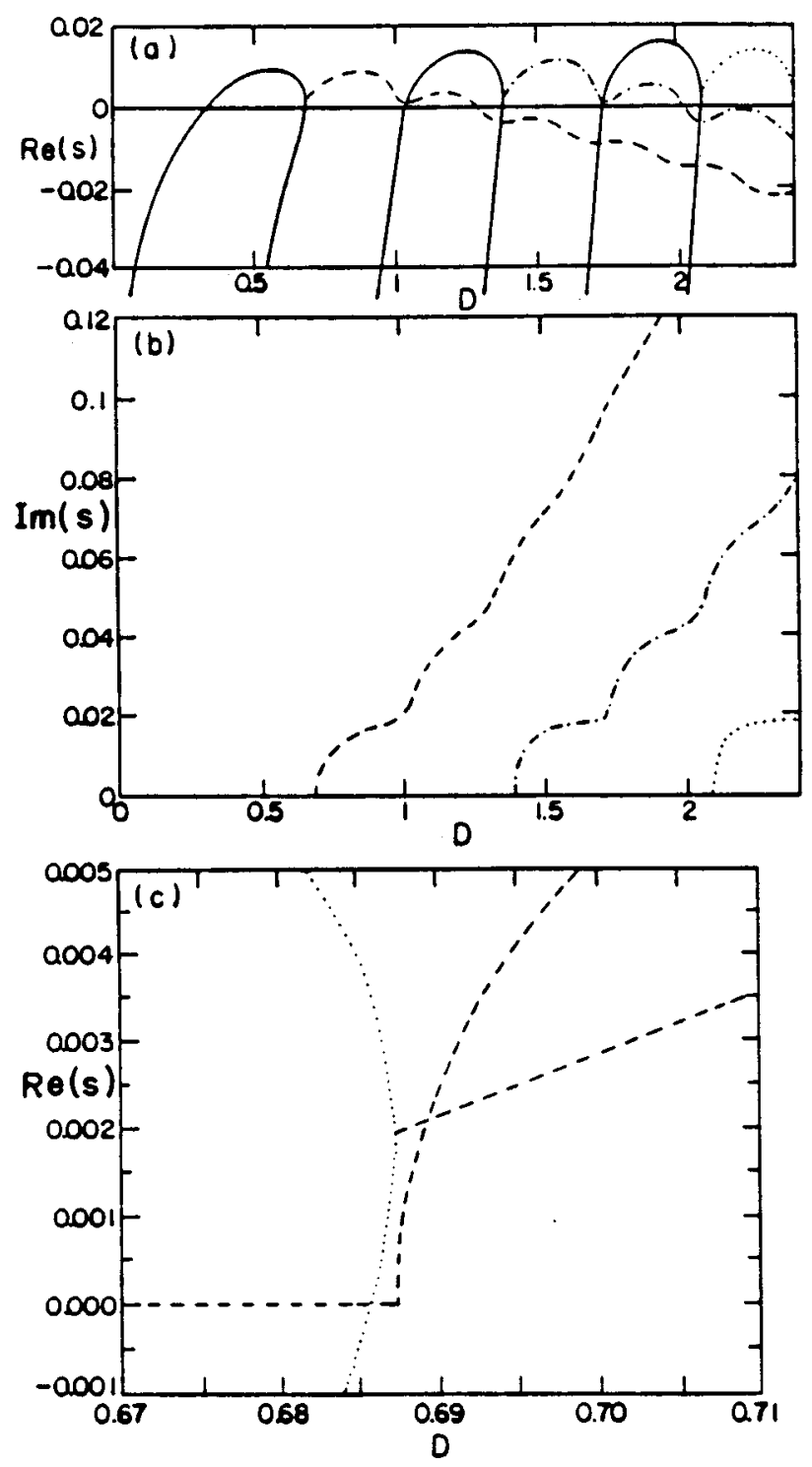

FIG. 4. Roots of the dispersion relation for $x_{i}=100$. (a) $\operatorname{Re}(s)$ as a function of $D$; (b) $\operatorname{Im}(s)$ as a function of $D$; (c) detail of (a).

the one indicated when describing Fig. 3(a), not clear in that figure because of the scale. Several features are qualitatively similar to those described in the previous cases. Note, however, that the stability islands are lost here, as one can realize observing the behaviour of the first oscillatory mode (dashed line) and the third monotonic mode (full line) in the range $D \approx 1$. The first and second oscillatory modes both show a second overstable interval, after the regions 3 and 5, respectively. The nondimensional growth rates are smaller than in the case $x_{i}=0.1$ roughly by a factor 100 .

The local maxima of the growth rates in each region, considered separately for monotonic and overstable modes, increase slowly as we move toward regions of higher order. This is a general trend present in all three $x_{i}$ cases studied.

\section{NONLINEAR STEADY STATES}

For a pair $\left(x_{i}, D\right)$, corresponding to a threshold, the system allows another possible linear steady state solution with nonuniform $v, n r$, and $\phi \neq 0$, besides the basic uniform solution $v=v_{b}, n r=n_{e} R_{e}$, and $\phi=0$. These nonuniform velocity states, which bifurcate from the basic solution at the critical values, are the linearized version of finite amplitude, steady state, spatially dependent potentials, that are also compatible with the equations of the model.

The importance of these states is due to the fact that numerical experiments show that some of them are asymptotic solutions, i.e., perturbations of the initial constant speed flow may evolve in time and relax to a nonlinear steady state. For planar diodes the stability of nonlinear states is studied in Refs. 8 and 9.

The nonlinear states are solutions of

$$
\frac{d}{d x}\left(x \frac{d}{d x} \psi\right)=\frac{D^{2}}{2}\left(\frac{1}{\sqrt{1+\psi}}-1\right),
$$

where $\psi=e \phi /\left(m v_{b}^{2} / 2\right), x=r / R_{e}$, with $\psi(1)=\psi\left(x_{i}\right)=0$. For $|\psi| \ll 1$ this equation is solved by the Bessel functions $J_{0}\left(D \sqrt{x_{i}}\right), Y_{0}\left(D \sqrt{x_{i}}\right)$. Using these solutions and boundary conditions it is easy to confirm, also in this way, that the linear critical states are given by the roots of (17).

The nonlinear steady state equation (18) follows from a Lagrangian variational principle,

$$
\delta \int_{1}^{x_{i}} \mathscr{L}\left(x, \psi, \psi^{\prime}\right) d x=0
$$

with

$$
\mathscr{L}\left(x, \psi, \psi^{\prime}\right)=\frac{x \psi^{\prime 2}}{2}+\frac{D^{2}}{2}(2 \sqrt{1+\psi}-\psi) .
$$

Thus (18) corresponds to

$$
\frac{\delta \mathscr{C}}{\delta \psi}=\frac{\partial \mathscr{C}}{\partial \psi}-\frac{\partial}{\partial x}\left(\frac{\partial \mathscr{C}}{\partial \psi^{\prime}}\right)=0 .
$$

Introducing, $\Pi \equiv \partial \mathscr{C} / \partial \psi^{\prime}=x \psi^{\prime}$, the canonical momentum associated with $\psi$, we obtain the Hamiltonian $\mathscr{H}=\Pi \psi^{\prime}-\mathscr{B}$,

$$
\mathscr{H}=\frac{\Pi^{2}}{2 x}+\frac{D^{2}}{2}(\psi-2 \sqrt{1+\psi}) .
$$

We may note that $\Pi^{2} / 2 x$ is the (nondimensional) electrostatic energy density of the system, here acting as equivalent kinetic energy in a mechanical analogy. The equivalent (nondimensional) potential energy density is $D^{2}(\psi-2 \sqrt{1+\psi}) / 2$.

From Hamilton's equations it follows directly that

$$
\frac{d \mathscr{H}}{d x}=-\frac{\Pi^{2}}{2 x} .
$$

Therefore $\mathscr{H}$ decreases with increasing $x$, and vice versa. Moreover, maxima or minima of the electrostatic potential $\psi$, are also maxima or minima of the Hamiltonian $\mathscr{H}$, considered as a function of $x$.

The mechanical analogue corresponds to the motion of a particle with variable mass $x$ in a one-dimensional potential well (see Fig. 5), where energy, varying according to (23), is not conserved. Mass is equal to 1 starting at the emitter, then increases linearly for diodes with $x_{i}>1$, or decreases linearly 


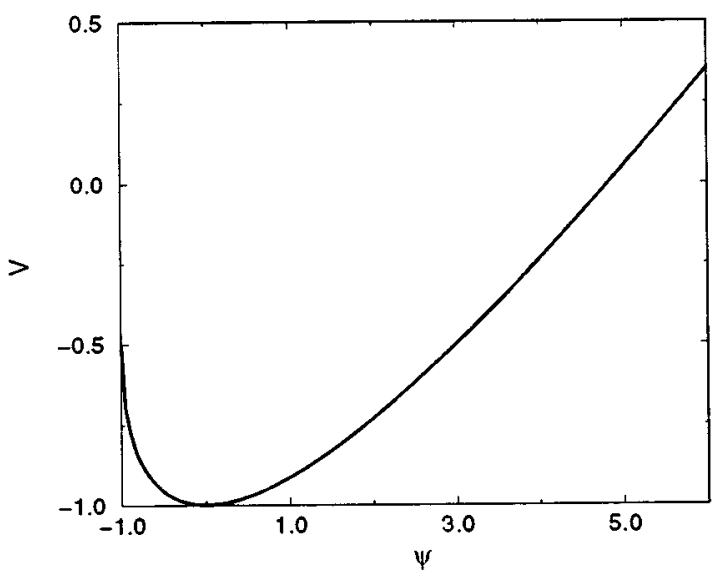

FIG. 5. Equivalent potential $V$ of the mechanical analogy versus $\psi$, for $D=1$.

for diodes with $x_{i}<1$. The fictitious particle is launched at $\psi=0$, with initial energy $\Pi^{2} / 2 x-D^{2} / 2$, and with $\Pi>0$ or $\Pi<0$, i.e., we may start with a negative or positive electric field at the emitter, respectively. After an excursion, with one or more passages through $\psi=0$, the particle may finally reach $\psi=0$, at the collector $\left(x_{i}\right)$. Thus $\psi$ may show one or more maxima, depending on the values of $\left(x_{i}, D\right)$. Of course, the coincidence of $\psi=0$ at the chosen final point $x_{i}$ depends on the starting conditions and corresponds to the solution of a characteristic value problem.

The principal limitation is the natural barrier at $\psi=-1$. This corresponds to the formation of a virtual cathode $(u=0, \nu \rightarrow \infty)$. The equations of the model fail at this point. Physically, the system becomes unstable when this value is attained by a solution. The mechanical analogy helps one to understand qualitatively the behaviour of different types of steady states. Solutions may have only one maximum of $\psi$ (one peak), one maximum and one minimum (a peak and a valley), two maxima (two peaks and a valley), and so on. Clearly, this depends on the number of oscillations the fictitious particle performs in the potential well, without touching the barrier.

By integration over $x$, we find $\mathscr{H}(1)>\mathscr{H}\left(x_{i}\right)$, when $x_{i}>1$, and $\mathscr{H}(1)<\mathscr{H}\left(x_{i}\right)$, when $x_{i}<1$. This implies that, while in planar diodes $\left|\psi^{\prime}\right|$ has the same value at emitter and collector, in cylindrical systems the steady state solutions must satisfy (taking boundary conditions into account) the inequalities $\psi^{\prime}(1)^{2}>x_{i} \psi^{\prime}\left(x_{i}\right)^{2}$, for $x_{i}>1$, and $\psi^{\prime}(1)^{2}$ $<x_{i} \psi^{\prime}\left(x_{i}\right)^{2}$, for $x_{i}<1$.

For a given value of $x_{i}$, nonlinear solutions can be obtained with an increasing number of peaks and valleys as $D$ increases. In region 1 there are solutions with one minimum. In region 2 we find solutions with one maximum, and solutions with one valley and one peak for higher values of $D$. Next, in region 3 for the divergent (convergent) case there are solutions with one peak, others with one valley and one peak for lower $D$ values, and solutions with two valleys (peaks) and one peak (valley) close to the limit with region 4.

This pattern repeats itself so that in the $2 n$th region one

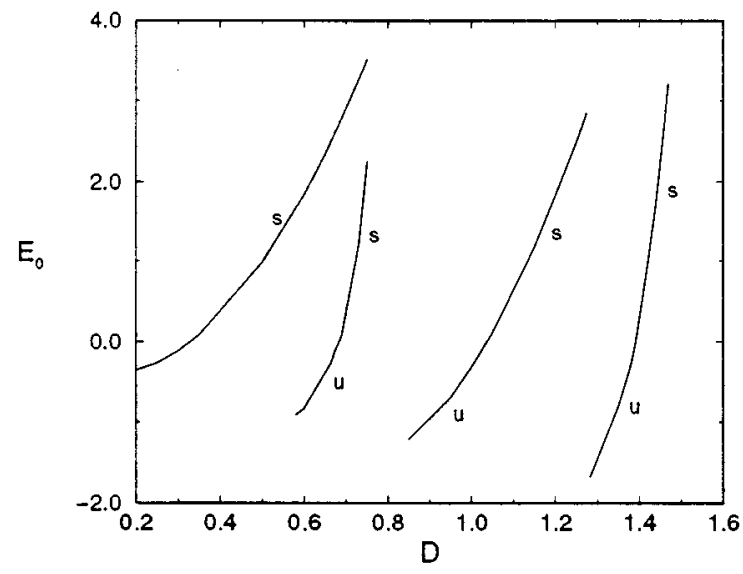

FIG. 6. Values of the electric field at the emitter, in nondimensional units, as a function of $D$, for the divergent case $x_{i}=100$.

would find solutions with one peak, others with $n$ peaks and $n-1$ valleys at the lower $D$ values, and a third group with $n$ valleys and $n$ peaks at the higher $D$ values, and in region $2 n+1$ solutions with one peak, others with $n$ peaks (valleys) and $n$ valleys (peaks) at the lower $D$ values, and a third group with $n$ valleys and $n-1$ peaks at the higher $D$ values.

These results have been confirmed numerically up to $D$ values in region 7 for $x_{i}=0.1,100$. Results are presented in Figs. 6 and 7 for the divergent and convergent case, respectively. We have plotted the values of the nondimensional electric field at the emitter $2 e R_{e} E_{r}(1) / m v_{b}^{2}$ vs $D$. The first branch, which corresponds to solutions with only one extremum, exists for all values of $D$. In region 1 the solutions are valleys, while in the rest of the regions these solutions are peaks. The value of the electric field at the emitter increases with $D$. A new branch corresponding to solutions with two peaks appears in the neighborhood of $D_{2}$, another one with three peaks in the neighborhood of $D_{3}$, and so on. The lowest and highest value of $D$ attainable in each branch are determined by virtual cathode formation. Examples of these

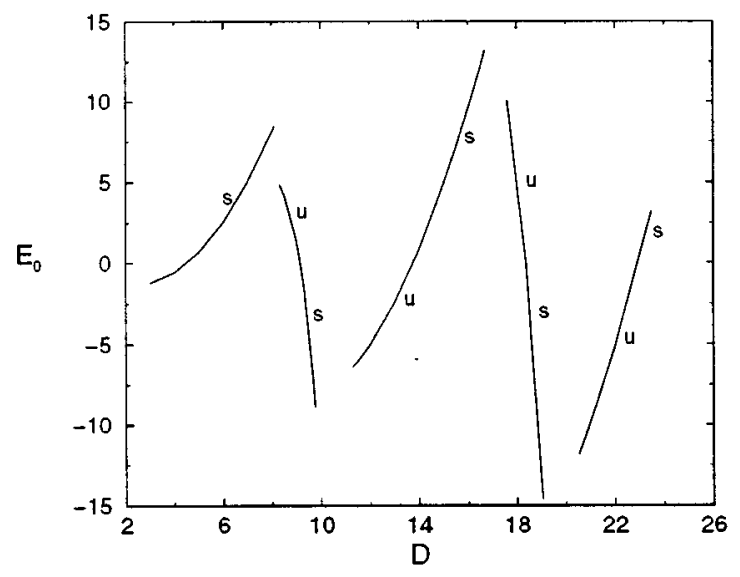

FIG. 7. Values of the electric field at the emitter, in nondimensional units, as a function of $D$, for the convegent case $x_{i}=0.1$. 


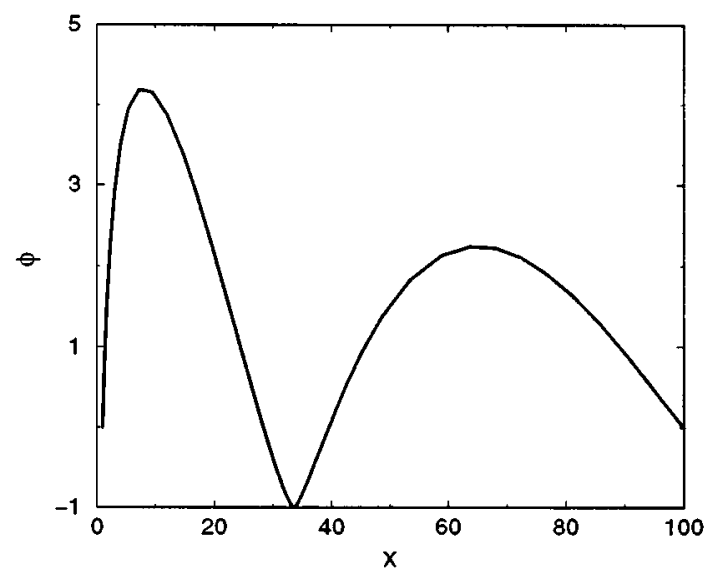

FIG. 8. Plot of the potential versus $x$ for $x_{i}=100$ and $D=1.275$. For $x=33.56$ the potential is -0.998 (close to virtual cathode formation).

limits are presented in Figs. 8 and 9 for the divergent and convergent cases, respectively.

The indications of stability of the different branches in Figs. 6 and 7 are given empirically, i.e., on the basis of the observed behaviour in numerical experiments. These stability properties are consistent as extensions of known results for planar diodes, but there are also new features. The branches around the values of $D_{n}$ with $n$ odd are similar to those found in the planar case. The new branches located in the neighborhood of $D_{n}$ with $n$ even, tend to the marginal modes of the planar case (see, Ref. 8) in the limit case $x_{i} \rightarrow 1$. In both situations, there is an exchange of stability between the zero potential solution and the nonuniform one as $D$ passes through $D_{n}$.

Considerable insight can be achieved by examining the simulations, taking into account concepts reported in the last two sections.

\section{PSEUDO-PLANAR DIODES}

When $x_{i} \approx 1$ the system is approximately a planar Pierce diode. As noted in Ref. 13 when $x_{i} \approx 1$ and $D \gg 1$, Eq. (17)

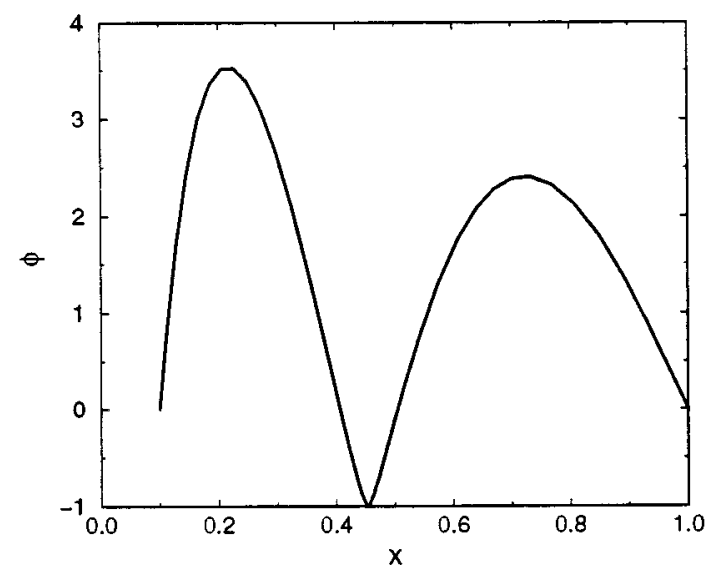

FIG. 9. Plot of the potential versus $x$ for $x_{i}=0.1$ and $D=16.7$. For $x=0.454$ the potential is -0.998 (close to virtual cathode formation).
TABLE I. Comparison of critical values for $x_{i}=1.3049$ and the planar case.

\begin{tabular}{crr}
\hline \hline \multicolumn{1}{c}{$\mathbf{D}$} & \multicolumn{1}{c}{$\alpha$} & $\alpha$ (planar) \\
\hline 22.0711 & 3.3647 & 3.1416 \\
44.1496 & 6.7306 & 6.2832 \\
66.2265 & 10.0962 & 9.4248 \\
88.3030 & 13.4618 & 12.5664 \\
110.3792 & 16.8273 & 15.7080 \\
\hline \hline
\end{tabular}

reduces to $\alpha=n \pi$, where $n=1,2,3, \ldots$, which is the condition for zero frequency roots for planar Pierce diodes. Also, $n$ defines the critical values that mark the boundaries between regions on the $\alpha$ line. The behaviour of the system for $x_{i}$ near 1 is then expected to show known properties of the planar Pierce diodes. In this section we show the results of a set of simulations of the cylindrical system for $x_{i}=1.3049$. These numerical experiments are therefore performed as a benchmark for the code, and are compared with existing particle simulations in the literature, in particular with Ref. 2. The linear dispersion relation is similar to figure 1 of Crystal and Kuhn. ${ }^{2}$ In fact, we can see in Table I how the critical $\alpha$ values for this cylindrical system are close to those of the planar case.

Computer experiments were prepared at $t=0$ with both an excess of electrons over ions, i.e., negative initialization, and a defect of electrons, i.e., positive initialization. This produces an initial nonzero electric potential. For $D<22.0711$ (region 1) the simulations show a stable behaviour in agreement with linear stability predicted by theory. The perturbation is found to decrease after a period of adjustment, and vanishes in a few transit times. Crossing the first critical value, the predicted linear instability in region 2 is observed, having the following nonlinear characteristics. First, for positive initialization we find that the electron flow does not become turbulent, but stays laminar. The electric potential increases and approaches an asymptotic steady state, which is a nonlinear solution with one maximum. The new state, with nonuniform velocity, appears to be stable in the simulation. An example of these features is shown in Fig. 10 , obtained for $D=33.0$ which corresponds approximately to the maximum linear growth rate in the region. Figure 10(a) shows the initial profile of the potential. Figure 10(b) shows the final shape of the potential. Note that the final amplitude is over two thousand times the initial value. Figure 10 (c) represents the asymptotic phase space picture (after 13 $\tau ; \tau$ : transit time) where we can see the change of the beam velocity across the diode. Finally, Fig. 10(d) shows the time evolution of the mid-potential (potential midway between emitter and collector), which grows and approaches a constant value after about $10 \tau$. No oscillations are observed. In accordance with the behaviour of the mid-potential, the number of particles in the simulation decreases, and finally becomes constant. Second, for negative initialization, the linear instability leads to the formation of a virtual cathode. Part of the electron beam is reflected toward the emitter, and part is transmitted through the diode. The virtual cathode is alternatively formed and disintegrated. There is no approach to a steady configuration. There is little particle trapping, so that 

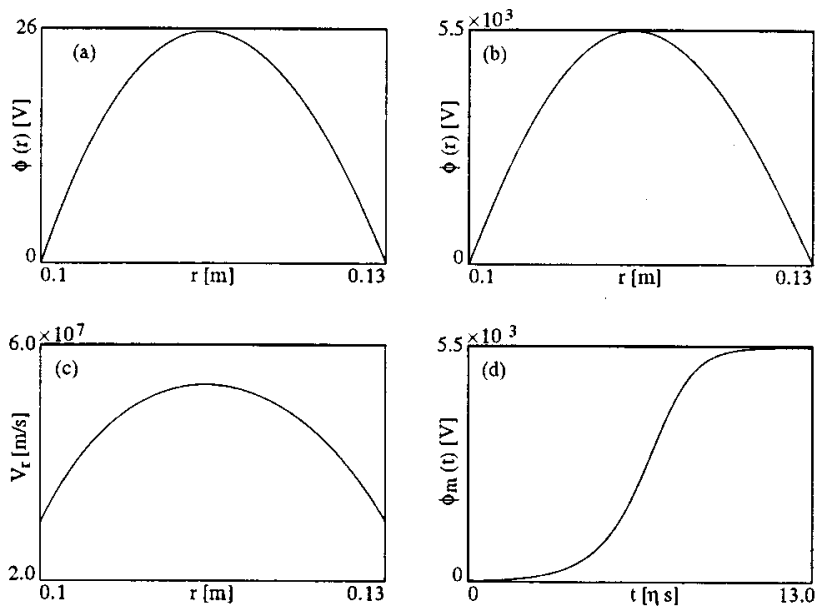

FIG. 10. Results for $x_{i}=1.3$ in region 2, with positive initialization: (a) initial potential profile; (b) final potential profile; (c) final phase space; (d) time evolution of mid-potential.

the instability is nonlinearly limited mainly by the formation of the virtual cathode, which implies an effective reduction of the interelectrode distance and electron current. Figure 11 shows the results of negative initialization for the same parameters of Fig. 10. In Fig. 11(a) we see the shape of the (negative) initial potential. Figure 11(b) represents the phase space at $t=13 \tau$ where a virtual cathode can be observed. Figure 11(c) gives the time evolution of the mid-potential, showing typical fluctuations of the system. Correspondingly, the number of particles of the simulation does not approach a constant value. These features are similar to those reported in Ref. 2 for region 2 of planar diodes.

Region 3 starts at $D=44.1496$. In this region the time asymptotic characteristics do not depend on the type of initialization (i.e., positive or negative). We find formation and disruption of a virtual cathode, and large amounts of trapped particles, as seen in the phase space plot of Fig. 12, for $t=12 \tau$ and $D=56$. This is similar to figure 16 (first bottom panel) of Ref. 2. In this region the electron flow is always
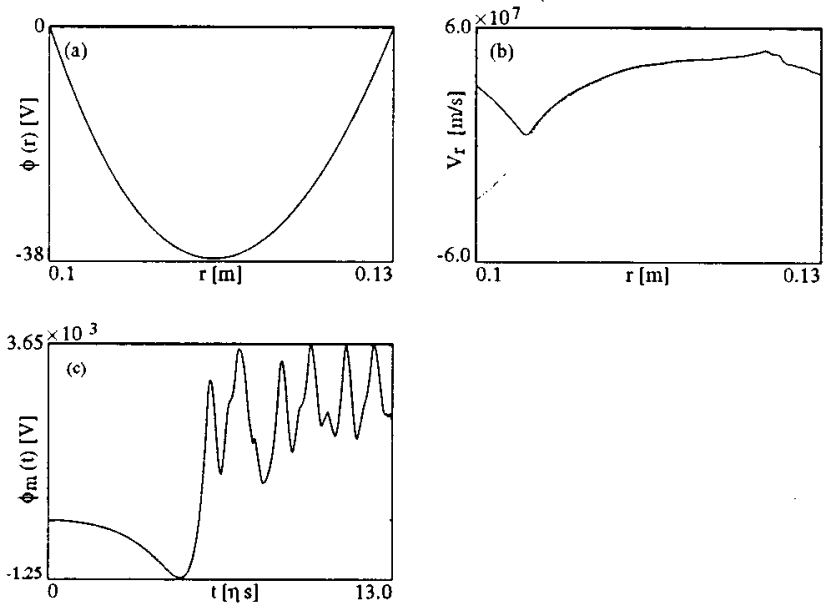

FIG. 11. Results for $x_{i}=1.3$ in region 2, with negative initialization: (a) initial potential profile; (b) final phase space; (c) time evolution of midpotential.

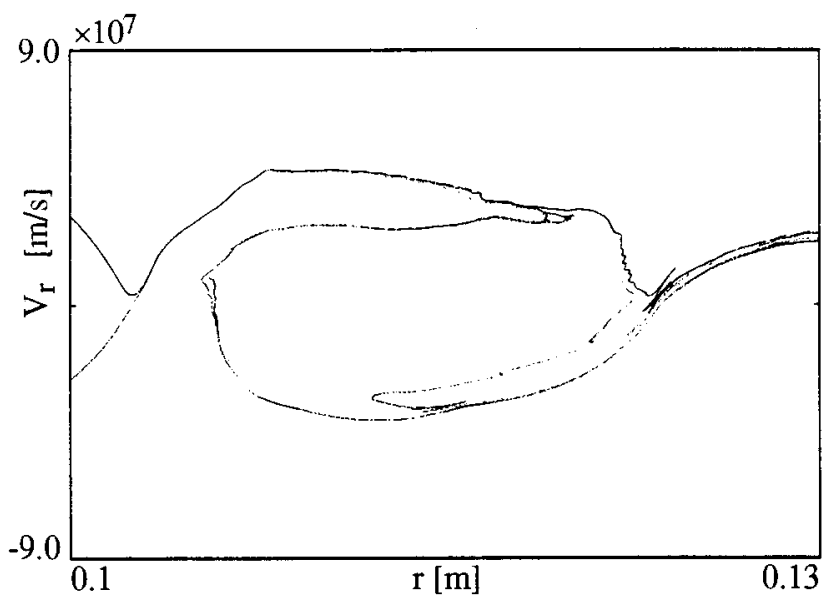

FIG. 12. Phase space for $x_{i}=1.3$ in region 3 , for $t=15 \tau$.

disrupted. The saturation of the linear instability is caused by virtual cathode formation and trapped particle processes.

For $D$ larger than 66.2265 we enter region 4 (not explored in Ref. 2). Here again, as in region 2, different behaviours of the computer experiments are obtained for positive and negative initialization. Starting with a positive potential at $t=0$, the linear instability which produces departure from the uniform velocity state is observed. However, the system preserves a laminar electron flow and relaxes toward a new equilibrium state characterized by an electric potential with two maxima. The asymptotic approach to this steady state (which is also a nonlinear solution) occurs now with oscillations (not present in region 2). All this can be seen in the example of Fig. 13 where $D=77.0$. We do not give here the initial potential which is similar in shape to Fig. 10(a). Figure 13(a) shows the asymptotic potential and Fig. 13(b) the phase space with the modulation of the velocity for $t=15 \tau$. Finally, Fig. 13(c) gives the time evolution of the midpotential where we observe oscillations about the asymptotic steady state. Small amplitude oscillations about the nonuniform equilibrium have an angular frequency $\omega \tau=3.8$. A
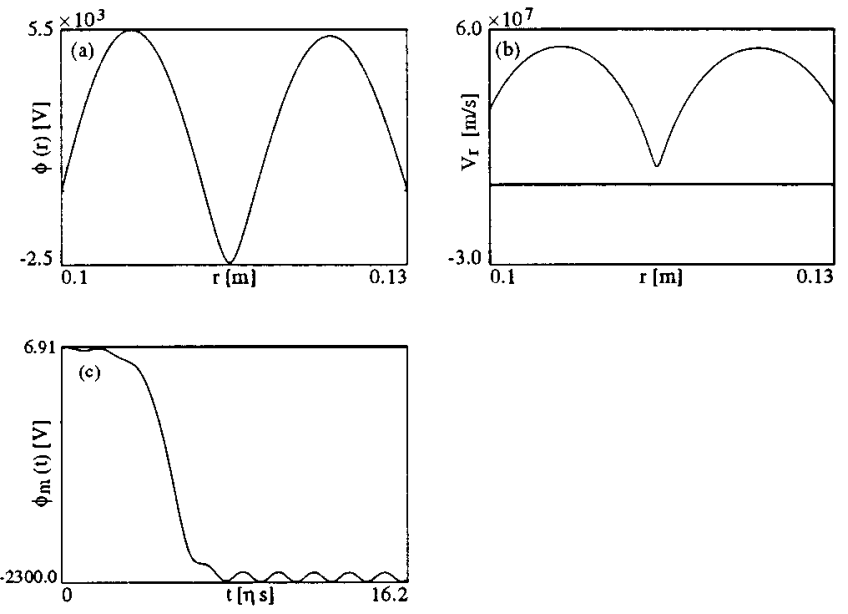

FIG. 13. Results for $x_{i}=1.3$ in region 4 , with positive initialization: (a) final potential profile; (b) final phase space; (c) time evolution of mid-potential. 

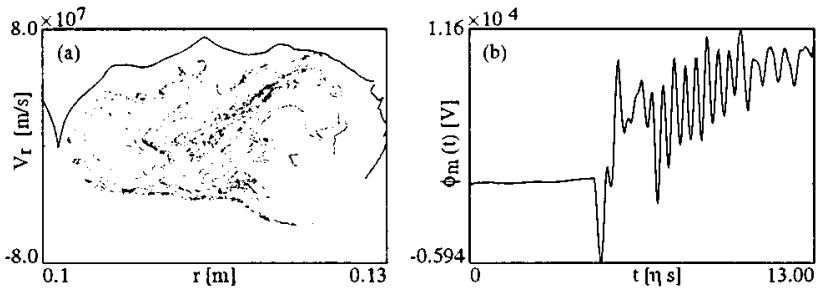

FIG. 14. Results for $x_{i}=1.3$ in region 4 , with negative initialization: (a) final phase space; (b) time evolution of mid-potential.

theory of this process for the planar case has been presented in Refs. 8 and 9. A detailed analysis of the dispersion relation for these modes can be found in Ref. 9 (see Eq. 40 and Fig. 12 in that reference) together with the numerical simulation of the pure oscillatory behaviour (Fig. 14 of the same reference). An approximate value of this pure oscillatory mode $(\alpha / \pi=3+3 / 8)^{9}$ is 3.7 , close to our measured frequency.

For negative initialization the flow is disrupted in a manner similar to region 3. This is exemplified in Fig. 14 for the same $D$ value as Fig. 13. We show in Fig. 14(a) the phase space, with a typical vortex and virtual cathode, at $t=13 \tau$ and in Fig. 14(b) the corresponding fluctuations of the midpotential. The number of particles also has a characteristic unsteady time behaviour.

The nonlinear behaviour reported in Ref. 2 for the planar diode in regions 1 to 3 has also been found in the $x_{i}=1.3049$ cylindrical case. Numerical experiments have been extended to regions 4 to 6 . In region 5 we have observed a behaviour similar to region 3 . In region 6 with positive initialization, the system relaxes to a nonlinear solution of the potential with three maxima. For negative initialization in region 6 the electron flow is disrupted. Summing up, the critical values defining the boundaries where the stability properties change have been verified.

\section{EFFECTS OF HIGHLY DIVERGENT FLOWS}

It is interesting to contrast now the behaviour of cylindrical diodes with large surface ratios $\left(x_{i} \gg 1\right)$, with the pseudo-planar case $\left(x_{i} \approx 1\right)$ of Sec. V. We studied in detail a system with $x_{i}=100$, so that the cylindrical geometry, the radial density variation, and the divergence of the flow should have a strong influence. The first six critical values for $D$ are $D_{1}=0.3314, \quad D_{2}=0.6858, \quad D_{3}=1.0377$, $D_{4}=1.3887, D_{5}=1.7390, D_{6}=2.0889$. We have confirmed by several computer experiments that the stability properties of the regions defined by these values change for $D$ below and above the boundary values.

Region 1 is stable in the simulations as predicted by theory. We have tried to destabilize the system with large (negative or positive) initializations with no consequence. The threshold of linear instability for this cylindrical diode is given by $\alpha=16.4043 \gg \pi$. Therefore, for the same interelectrode distance and beam velocity, the current density threshold at the emitter is 27 times higher than in the planar diode. Results for region 2 are given for $D=0.5$ in Fig. 15. The
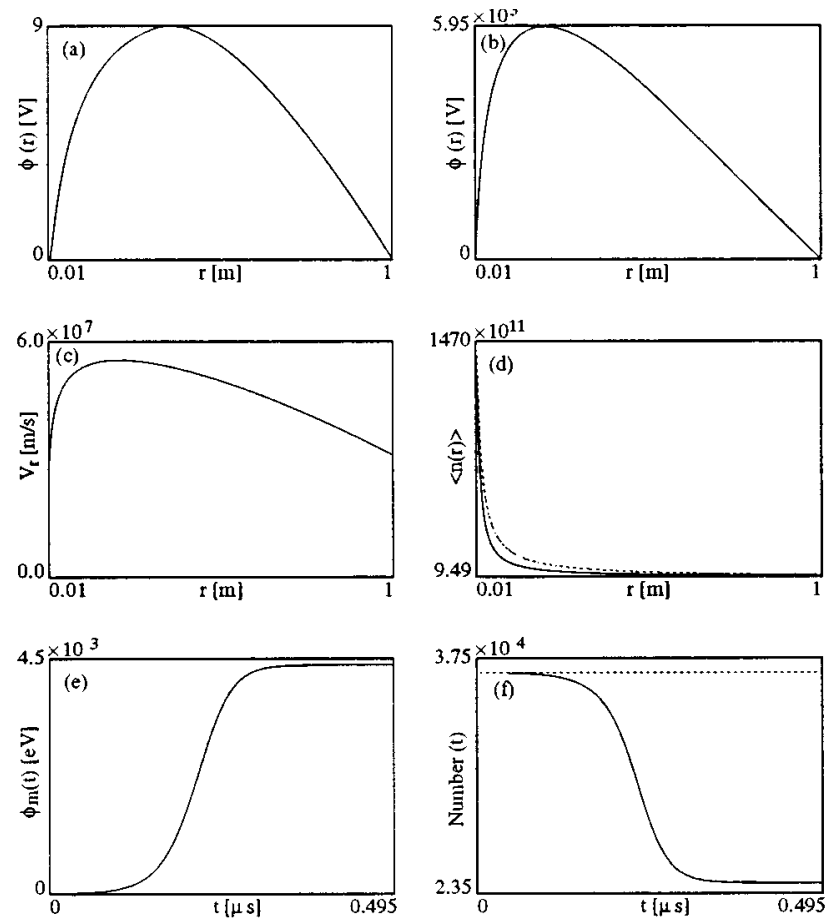

FIG. 15. Results for $x_{i}=100$ in region 2, with positive initialization: (a) initial potential profile; (b) final potential profile; (c) final phase space; (d) time average of density; (e) time evolution of mid-potential; (f) time evolution of number of particles.

initial (positive) potential is presented in Fig. 15(a). The linear instability develops and takes the system to a new (nonuniform) equilibrium shown in Fig. 15(b) at $t=15 \tau$. The potential maximum (four orders of magnitude larger than the initial value) is clearly shifted toward the emitter, and the phase space, Fig. 15(c), shows a similar asymmetry. The maximum velocity is nearly two times $v_{b}$. We give in Fig. 15 (d) the density profile of electrons averaged over two transit times around $t=19 \tau$ (solid line). Note the difference with the ions (dotted line) showing that the system has a net positive charge throughout. Figure 15(e) gives the time evolution of the number of particles showing about a $30 \%$ decrease from $t=0$ to equilibrium. Figure 15(f) shows the approach to the asymptotic equilibrium via the time evolution of the midpotential. No oscillations are observed as the constant final value is achieved.

The behaviour of the divergent flow with $x_{i}=100$ in regions 3 and 5, or in regions 2, 4 and 6 with negative initialization, is most unusual. The nonlinear stage shows a strong dynamics, with formation and coexistence of several transient and moving virtual cathodes in the interelectrode space. The accelerating potential of moving virtual cathodes is a phenomenon characteristic of high surface ratio diodes, not present at small ones. The formation of several jets of electrons can be observed at a given time at different positions through the system (five can often be seen, with speeds up to two times $v_{b}$ ). The jets are generated by the moving virtual cathodes in a kind of "ejection" mode, while parts of the beam are reflected backward. Remarkably, there is very little particle trapping in this regime. The growth of the elec- 

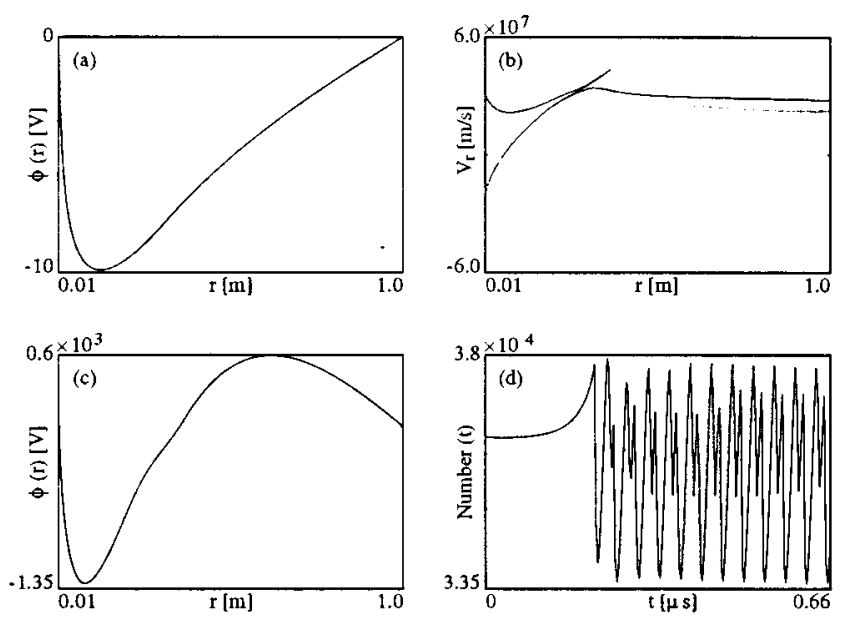

FIG. 16. Results for $x_{i}=100$ in region 2, with negative initialization: (a) initial potential profile; (b) final phase space; (c) final potential profile; (d) time evolution of number of particles.

trostatic energy in the instability is limited mainly by conversion to multiple energetic electron jets. The system keeps going in this strongly fluctuating fashion during very long simulation runs. Examples of these processes are illustrated in the following.

The electron flow can be disrupted in region 2 when negative initialization is used. For the same value of $D=0.5$ this phenomenon is illustrated in Fig. 16. In Fig. 16(a) we give the initial potential. A picture of phase space is shown in Fig. 16(b) for $t=20 \tau$, where one can see the reflected particles but also the formation of a jet of forward accelerated particles. The corresponding electric potential profile is shown in Fig. 16(c) and the fluctuations of the number of particles around the initial value in Fig. 16(d). First appearance of a virtual cathode occurs at about $t=10 \tau$. The position of the virtual cathode travels away from the emitter. The time-varying potential energizes a set of particles. Comparing Fig. 11(b) of the pseudo-planar diode with the present phase space [Fig. 16(b)] we can perceive the monotonic deceleration of the beam from emitter to virtual cathode in the former case, in contrast with deceleration followed by acceleration of the latter. As the virtual cathode approaches the collector, it fades away, but a new one forms near the emitter and another cycle starts. A signature of the repetition of this process is given by the oscillations shown in Fig. 16(d). Little particle trapping is observed. From time to time the collector receives two sets of particles with well defined energies.

In regions 3 and 5 the system is disrupted by the instability with both kinds of initialization. In Fig. 17(a) we show the phase space of region 3 for $t=15 \tau$ and $D=0.87$. The initialization potential was positive with a maximum of about $5 \mathrm{~V}$. A recently formed accelerating virtual cathode and an old one near the collector are both visible. The time history of the mid-potential is shown in Fig. 17(b). The phase space for $D=1.6$ (region 5) is presented in Fig. 17(c) at $t=44 \tau$. The initialization was positive with a maximum near 15 V. Several accelerating potentials can be seen. Cor-
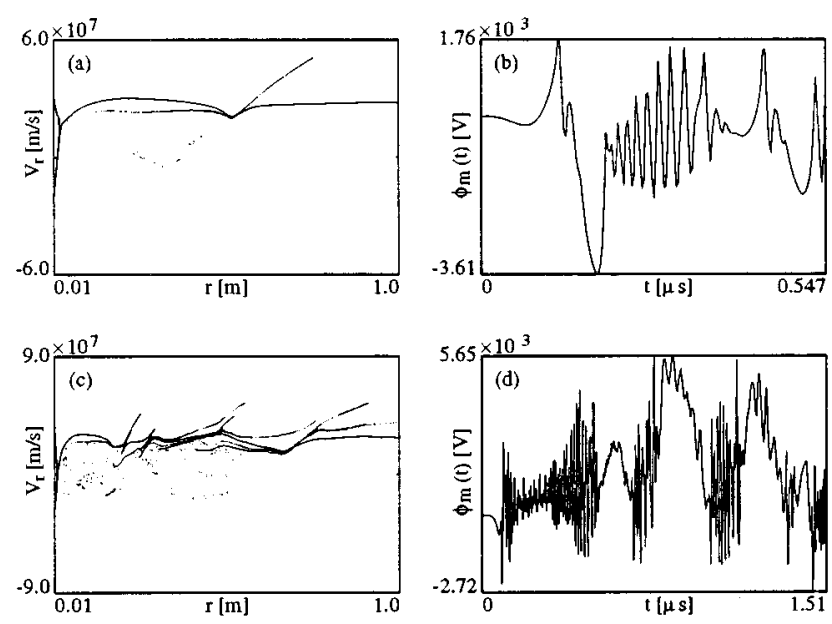

FIG. 17. Results for $x_{i}=100$ in region 3 [(a) and (b)] and in region 5 [(c) and (d)]: (a) final space phase; (b) time evolution of mid-potential; (c) final phase space; (d) time evolution of mid-potential.

respondingly, the fluctuations of the mid-potential are given in Fig. 17(d).

Region 4 is characterized by the fact that positive initialization leads to a new nonuniform equilibrium state, while negative initialization produces a disruption of the electron flow. We show in Fig. 18(a) an example of asymptotic potential with two maxima, for $D=1.2$, attained at time of order $10 \tau$. Correspondingly, Fig. 18(b) shows the phase space. The time averaged electron density (over the last two transit times) is represented by a solid line in Fig. 18(c) (the dotted line is the ion density). There is a net positive charge density with the exception of a region around the minimum of the electric potential. The mid-potential, Fig. 18(d), exhibits oscillations as the asymptotic state is attained. These oscillations with frequency $\omega \tau=3.33$ may be associated with small amplitude perturbations of the nonlinear equilibrium (not yet analyzed for cylindrical diodes).
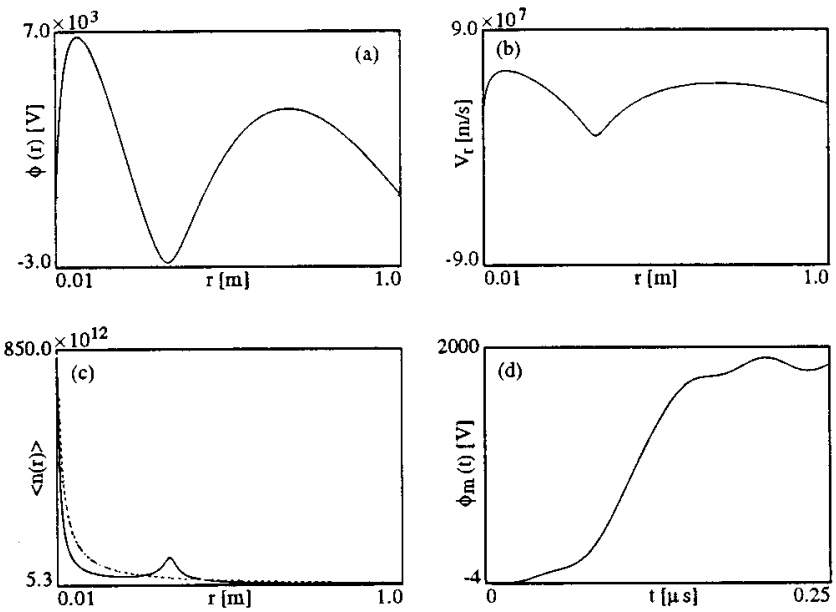

FIG. 18. Results for $x_{i}=100$ in region 4, with positive initialization: (a) final potential profile; (b) final phase space; (c) time average density; (d) time evolution of mid-potential. 

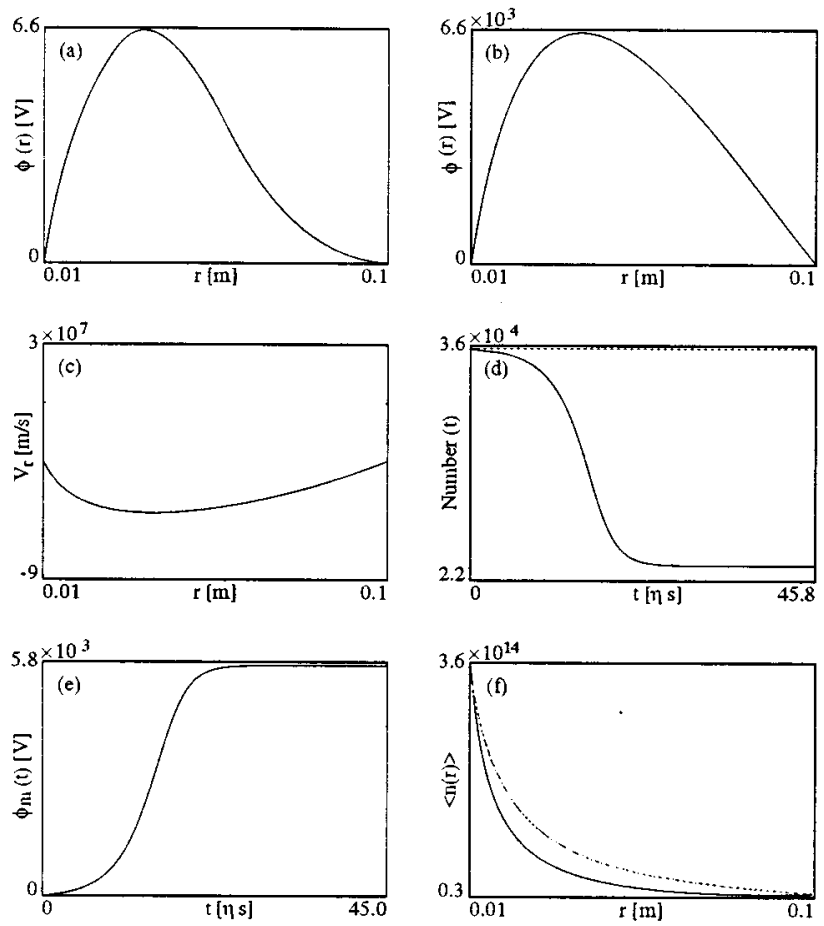

FIG. 19. Results for $x_{i}=0.1$ in region 2, with positive initialization: (a) initial potential profile; (b) final potential profile; (c) final phase space; (d) time evolution of number of particles; (e) time evolution of mid-potential; (f) time average of density.

\section{STRONGLY FOCUSED BEAMS}

As mentioned in the introduction the convergent configuration $\left(x_{i}<<1\right)$ is the most interesting one for applications in which ions are neutralized with comoving electrons. We shall describe the main features of this case by computer experiments for a particular value of the ratio $x_{i}=0.1$, which corresponds to a strong convergence of the beams.

The first critical $D$ values for $x_{i}=0.1$ are $D_{1}=4.5232$, $D_{2}=9.1487, \quad D_{3}=13.7557, \quad D_{4}=18.3569, \quad D_{5}=22.9555$, and $D_{6}=27.5528$. Region 1 is stable in computer experiments, as in the previous cases studied in Secs. V and VI. The first threshold of the instability corresponds to $\alpha=2.0354$, so that for the same interelectrode distance and velocity of the beams the density current at the emitter for this cylindrical case is nearly 2.4 times smaller than in the planar diode. Simulations show that the second region is linearly unstable. However, a new feature appears in the nonlinear evolution of the system. Positive initializations lead to a new nonuniform equilibrium, as we have also found for $x_{i}=1.3$ and $x_{i}=100$. For negative initialization, instead, we must distinguish between "small" and "large" initial perturbations. For the time being this is only a qualitative characterization of the initial perturbation strength to be determined empirically. For small negative perturbations the system relaxes to a nonuniform equilibrium and only for large negative perturbations is the system disrupted.

We present first an example of positive initialization for region 2 , corresponding to $D=7.0$ close to the maximum growth rate. In Fig. 19(a) we show the initial potential. Figure 19 (b) is the asymptotic potential for $t \approx 14 \tau$. The phase
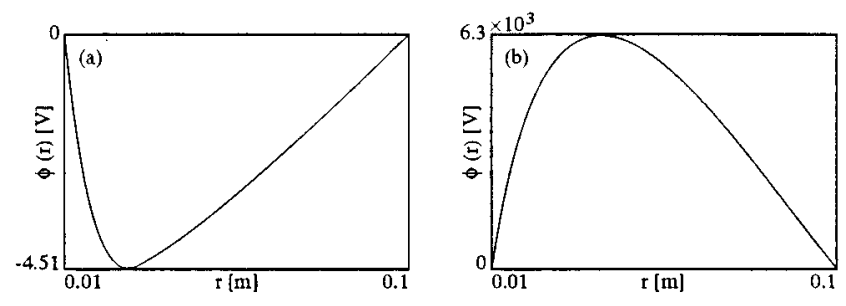

FIG. 20. Results for $x_{i}=0.1$ in region 2, with a small negative initialization: (a) initial potential profile; (b) final potential profile.

space, at the same time, is in Fig. 19(c). We may note that the maximum of the potential as well as the maximum of the electron speed is here shifted toward the collector, in contrast to the case of $x_{i}=100$, where the maxima were shifted toward the emitter. In Figs. 19(d) and 19(e) we can follow the relaxation to the new equilibrium via the number of particles and the mid-potential as a function of time, respectively. No oscillations are observed. Finally, in Fig. 19(f) we give the time averaged electron density (solid line, averaged over 7 transit times) where the ion density is also given as reference (dotted line).

We examine now two negative initializations of different strength for region 2 and the same $D=7$. In Fig. 20(a) we show the initial potential with a maximum amplitude not exceeding $4.5 \mathrm{~V}$ in absolute value. The system evolves to a nonlinear equilibrium solution for the potential in about $10 \tau$ as shown in Fig. 20(b). Note that this is the same potential reported by Fig. 19(b). The number of particles and midpotential evolutions are quite similar to those reported in Figs. 19(c) and 19(d). The same can be said about the asymptotic phase space. However, if the initial perturbation is larger, as shown in Fig. 21(a), where the maximum of $|\phi| \simeq 13 \mathrm{~V}$, the flow is disrupted in about $10 \tau$. Figure 21(b) shows the phase space shortly after the formation of the virtual cathode, where reflection of particles and also the trapping of another group of particles near the collector can be seen. Figure 21(c) shows the phase space at $t \simeq 25 \tau$ and Fig.
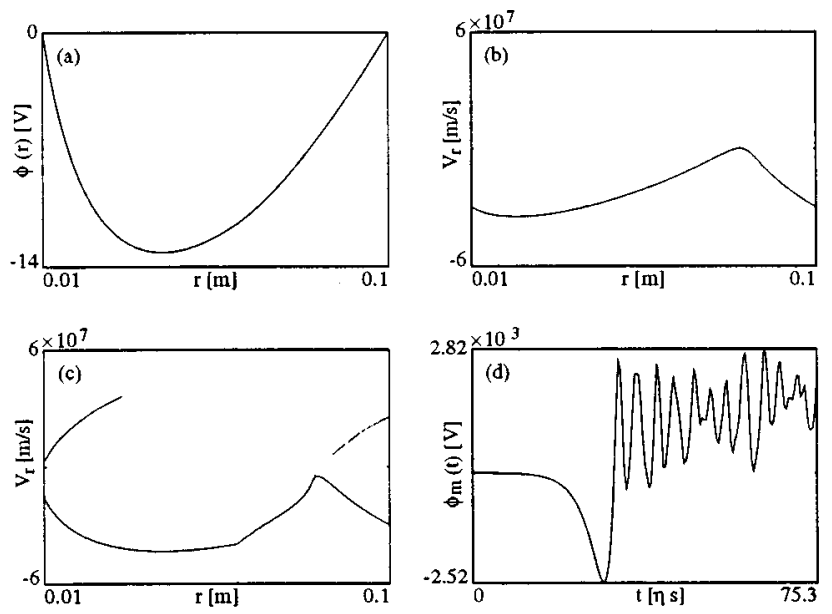

FIG. 21. Results for $x_{i}=0.1$ in region 2 with a large negative initialization: (a) initial potential profile; (b) phase space just after the virtual cathode formation; (c) phase space at $t=25 \tau$; (d) time evolution of mid-potential. 

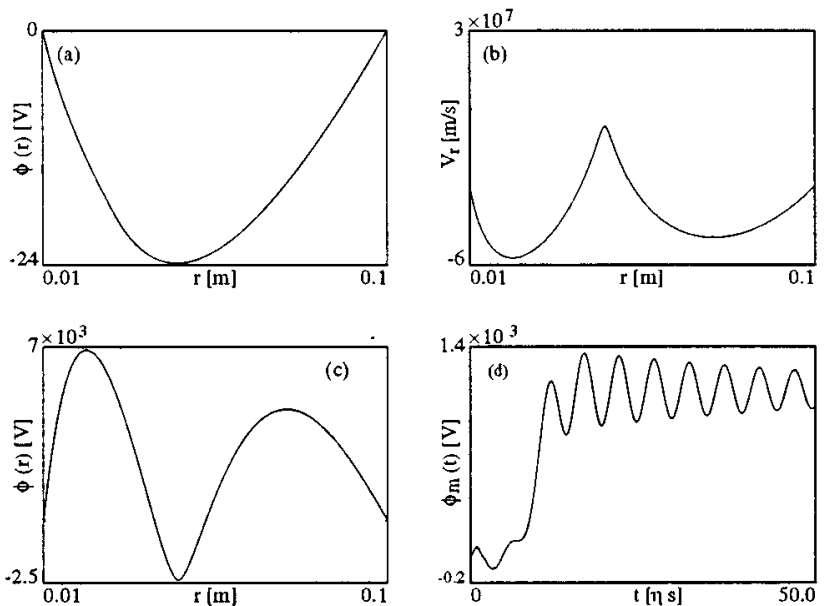

FIG. 22. Results for $x_{i}=0.1$ in region 4 , with a small negative initialization: $|\phi| \simeq 24 \mathrm{~V}$ : (a) initial potential profile; (b) phase space at $t=17 \tau$; (c) potential profile $t=17 \tau$; (d) time evolution of mid-potential.

21(d) gives the history of the mid-potential. Here the instability is limited mainly by formation of the virtual cathode.

In region 4 this behaviour is more pronounced. For positive initialization the system attains a new nonuniform equilibrium with a two peak potential. The electron flow remains laminar. Small negative initialization leads to a nonlinear stable solution, while large negative initialization disrupts the electron flow. In Fig. 22(a) we show the initial negative potential with a maximum $|\phi| \simeq 24 \mathrm{~V}$, for the case $D=16$ (close to the maximum growth rate of region 4). Figure 22(b) shows the asymptotic phase space at $t \simeq 17 \tau$, and Fig. 22(c) the corresponding potential. The approach to the new equilibrium takes place with weakly damped oscillations revealed by the mid-potential time variation shown in Fig. 22(d). Measuring the angular frequency of the oscillations we find $\omega \tau=3.7$. These oscillations we ascribe again to small amplitude perturbations of the nonlinear equilibrium.

We see in Fig. 23(a) the potential corresponding to a
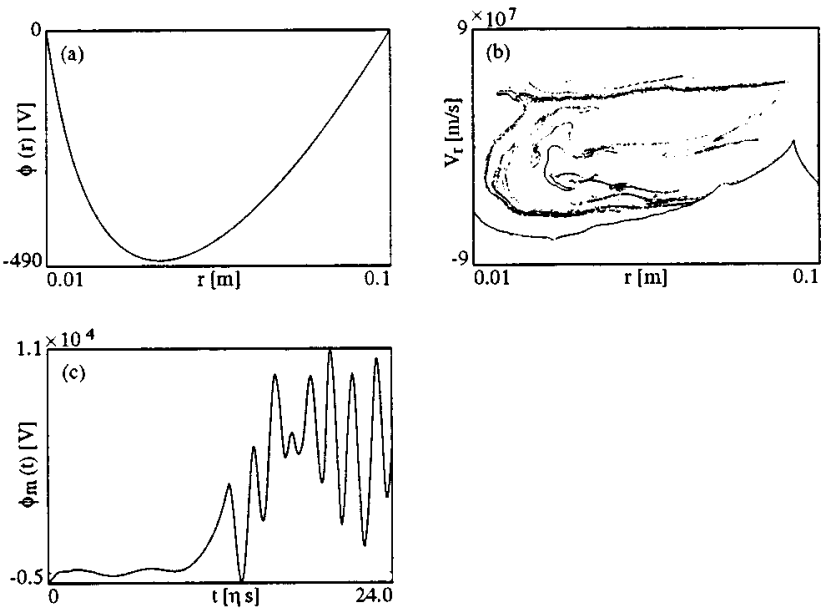

FIG. 23. Results for $x_{i}=0.1$ in region 4, with a large negative initialization: $|\phi| \simeq 490 \mathrm{~V}$ : (a) initial potential profile; (b) phase space at $t=8 \tau$; (c) time evolution of mid-potential.
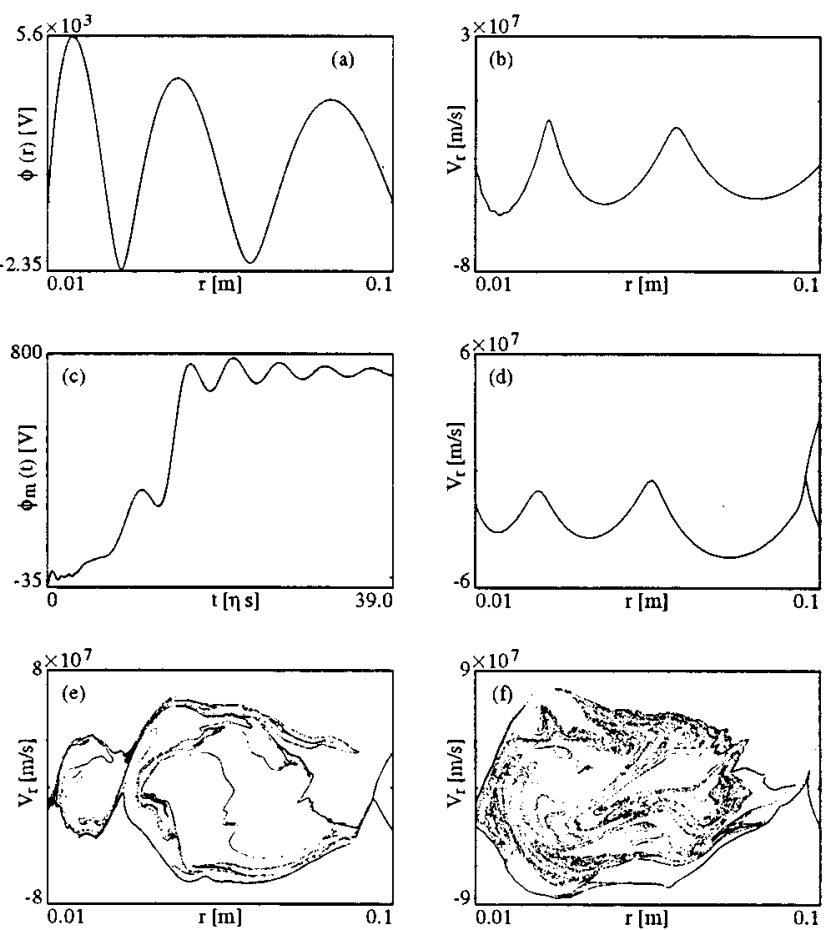

FIG. 24. Results for $x_{i}=0.1$ in region 6 , with two different negative initialization: $|\phi| \simeq 35 \mathrm{~V}$ (a)-(c), and $|\phi| \simeq 359 \mathrm{~V}$ (d)-(f). (a) Potential profile at $t=13 \tau$; (b) phase space at $t=13 \tau$; (c) time evolution of mid-potential; (d) phase space at $t \simeq 4 \tau$; (e) phase space at $t \simeq 6 \tau$; (f) phase space at $t \simeq 10 \tau$.

larger perturbation (negative initialization) with $|\phi| \simeq 490 \mathrm{~V}$ again using $D=16$. Figure 23 (b) shows the phase space at $t \simeq 8 \tau$. One can observe the virtual cathode and a large number of trapped particles. Figure 23(c) shows the fluctuations of the mid potential. Thus a larger negative initialization produces the disruption of the system.

The same qualitative behaviour is also found in region 6 . We give here examples for $D=25$. Figures 24(a) and 24(b) show the phase space and corresponding potential with three peaks, respectively, after $13 \tau$, for a small negative initialization (maximum of $|\phi|=177 \mathrm{~V}$ ). In Fig. 24(c) we can observe the oscillations of the mid-potential with an angular frequency $\omega \tau=3.7$, associated as before with perturbations of the nonlinear state. With a larger negative initialization (maximum of $|\phi|=358 \mathrm{~V}$ ) the system is disrupted in about $5 \tau$ as we can see in the phase space in Fig. 24(d), where a virtual cathode has just formed. Figures 24(e)-(f) show the phase space at later times, $t=6.1 \tau$ and $t=10 \tau$, respectively.

Let us return now to regions 3 and 5, which turn to be unstable for both initializations. As an example of the disruptions we present results for region 3 with $D=11.5$. Figures 25(a)-(d) show the phase space for times 10,12, 15 and $20 \tau$, respectively. Note that the first reflection of particles occurs near the collector but the virtual cathode is formed near the emitter.

\section{DISCUSSIONS AND CONCLUSIONS}

In this paper we study the electron instability and the nonlinear behaviour of cylindrical Pierce diodes with radial 

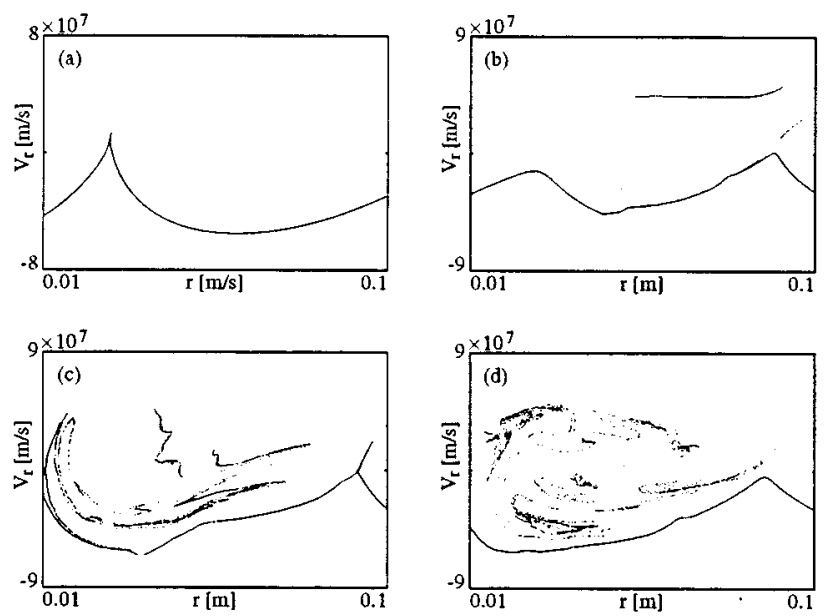

FIG. 25. Results for $x_{i}=0.1$ in region 3: (a) phase space at $t=10 \tau$; (b) phase space at $t=12 \tau$; (c) phase space at $t=15 \tau$; (d) phase space at $t=20 \tau$.

flow. The linear properties of these systems depend on two parameters, $x_{i}$ and $D$ (or $\alpha$ ). We have given detailed solutions for the linear dispersion relation in the cases $x_{i}=0.1$, $x_{i}=3.1765$, and $x_{i}=100$. The first and last cases are then studied in the nonlinear stage with numerical experiments. Critical values of $D$, for which $s=0$, given by Eq. (17), define regions where the system can be nonlinearly stable or unstable depending on initial conditions of space charge. For small values of $x_{i}$, intervals of stability appear close to $D_{3}$, $D_{5}, \ldots$, etc. (stability islands). These small stable regions are not present in systems with large values of $x_{i}$.

We found that the alternating succession of oscillatory unstable and monotonically unstable modes that appear in the system as $D$ increases, is different in systems with $x_{i}<1$ and $x_{i}>1$, compared to the planar case. For instance, Fig. 4(a), for $x_{i}=100$, shows that the coalescence of two pure imaginary roots into a complex conjugate pair (overstable mode) occurs for a value of $D$ somewhat larger than $D_{2}$. Hence, with increasing $D$ a new monotonically unstable mode appears at $D_{2}$, and afterwards the overstability appears inside region 3 as a consequence of the merging of two monotonically growing modes. A similar pattern can be seen near $D_{4}, D_{6}, \ldots$, etc., and it takes place also for $x_{i}<1$. In planar diodes the coalescence takes place exactly at $D_{2}$, $D_{4}, \ldots$, etc.

This feature of small amplitude theory is associated with changes of stability properties of the nonlinear steady states that bifurcate at the critical $D$ values, with respect to the behaviour characteristic of planar diodes. In Sec. IV we have shown the modifications that the so-called marginal states of planar systems undergo in cylindrical systems. ${ }^{8,9}$ In the amplitude vs $D$ diagrams of cylindrical diodes, an exchange of stability of nonlinear steady states, occurs at $D_{2}, D_{4}, \ldots$, etc. For $x_{i}<1$, and with increasing $D$, we pass from unstable to stable nonlinear states. Conversely, for $x_{i}>1$, stable turn into unstable nonlinear states. These features may have interesting consequences in the analysis of bifurcations and chaotic behaviour. A deeper investigation of these topics is matter for future studies.
TABLE II. Main results. $\mathrm{S}=$ stable; $\mathrm{U}=$ unstable; $\mathrm{NLS}=$ nonlinearly stable; $\mathrm{VC}=$ virtual cathode; $(\mathrm{N}) \mathrm{TP}=($ no $)$ trapped particles; $(\mathrm{N}) \mathrm{O}=($ no $)$ oscillations; $\mathrm{J}=$ jets; $\mathrm{N}(\mathrm{P}) \mathrm{I}=$ negative (positive) initialization; $\mathrm{S}(\mathrm{L}) \mathrm{NI}=$ small (large) negative initialization; $\# \mathrm{P}=$ number of peaks in the NLS solution.

\begin{tabular}{|c|c|c|c|c|c|c|}
\hline Regions & 1 & 2 & 3 & 4 & 5 & 6 \\
\hline \multicolumn{7}{|l|}{1.3} \\
\hline$D_{i}=$ & 22.071 & 44.149 & 66.226 & 88.303 & 110.379 & \\
\hline \multirow[t]{3}{*}{$\mathrm{PI} \rightarrow$} & $\mathbf{S}$ & NLS & $\mathbf{U}$ & NLS & $\mathbf{U}$ & NLS \\
\hline & & $1 \mathrm{P}$ & $\mathrm{O}, \mathrm{VC}$ & $2 \mathrm{P}$ & $\mathrm{O}, \mathrm{VC}$ & $3 \mathrm{P}$ \\
\hline & & $\mathrm{NO}$ & $\mathrm{TP}$ & $\mathrm{O}$ & TP & $\mathrm{O}$ \\
\hline \multirow[t]{3}{*}{$\mathrm{NI} \rightarrow$} & $\mathbf{S}$ & $\mathbf{U}$ & $\mathbf{U}$ & $\mathbf{U}$ & $\mathbf{U}$ & $\mathbf{U}$ \\
\hline & & $\mathrm{O}, \mathrm{VC}$ & $\mathrm{O}, \mathrm{VC}$ & $\mathrm{O}, \mathrm{VC}$ & $\mathrm{O}, \mathrm{VC}$ & $\mathrm{O}, \mathrm{VC}$ \\
\hline & & NTP & $\mathrm{TP}$ & $\mathrm{TP}$ & $\mathrm{TP}$ & $\mathrm{TP}$ \\
\hline \multicolumn{7}{|l|}{100} \\
\hline$D_{i}=$ & 0.331 & 0.686 & 1.038 & 1.289 & 1.739 & 2.089 \\
\hline \multirow[t]{3}{*}{$\mathrm{PI} \rightarrow$} & $\mathbf{S}$ & NLS & $\mathbf{U}$ & NLS & $\mathbf{U}$ & NLS \\
\hline & & $1 \mathrm{P}$ & $\mathrm{O}, \mathrm{VC}$ & $2 \mathrm{P}$ & $\mathrm{O}, \mathrm{VC}$ & $3 P$ \\
\hline & & $\mathrm{NO}$ & $\mathrm{TP}, \mathrm{J}$ & & $\mathrm{TP}, \mathrm{J}$ & $\mathrm{O}$ \\
\hline \multirow[t]{3}{*}{$\mathrm{NI} \rightarrow$} & $\mathbf{S}$ & $\mathbf{U}$ & $\mathbf{U}$ & $\mathbf{U}$ & $\mathbf{U}$ & $\mathbf{U}$ \\
\hline & & $\mathrm{O}, \mathrm{VC}$ & $\mathrm{O}, \mathrm{VC}$ & $\mathrm{O}, \mathrm{VC}$ & $\mathrm{O}, \mathrm{VC}$ & $\mathrm{O}, \mathrm{VC}$ \\
\hline & & NTP & $\mathrm{TP}, \mathrm{J}$ & $\mathrm{TP}, \mathrm{J}$ & $\mathrm{TP}, \mathrm{J}$ & $\mathrm{TP}, \mathrm{J}$ \\
\hline \multicolumn{7}{|l|}{0.1} \\
\hline$D_{1}=$ & 4.523 & 9.149 & 13.756 & 18.357 & 22.956 & 27.553 \\
\hline $\mathrm{PI} \rightarrow$ & $\mathbf{S}$ & NLS & $\mathbf{U}$ & NLS & $\mathbf{U}$ & NLS \\
\hline \multirow[t]{2}{*}{ or SNI } & & $1 \mathrm{P}$ & $\mathrm{O}, \mathrm{VC}$ & $2 \mathrm{P}$ & $\mathrm{O}, \mathrm{VC}$ & $3 \mathrm{P}$ \\
\hline & & $\mathrm{NO}$ & TP & $\mathrm{O}$ & $\mathrm{TP}$ & $\mathrm{O}$ \\
\hline \multirow[t]{3}{*}{$\mathrm{LNI} \rightarrow$} & $\mathbf{S}$ & $\mathbf{U}$ & $\mathbf{U}$ & $\mathbf{U}$ & $\mathbf{U}$ & $\mathbf{U}$ \\
\hline & & $\mathrm{O}, \mathrm{VC}$ & $\mathrm{O}, \mathrm{VC}$ & $\mathrm{O}, \mathrm{VC}$ & $\mathrm{O}, \mathrm{VC}$ & $\mathrm{O}, \mathrm{VC}$ \\
\hline & & NTP & $\mathrm{TP}$ & $\mathrm{TP}$ & $\mathrm{TP}$ & $\mathrm{TP}$ \\
\hline
\end{tabular}

The particle simulations of cylindrical Pierce diodes with radial flow have successfully recovered the predicted linear properties. Results of experiments for the case we have called pseudo-planar $\left(x_{i} \simeq 1.3\right)$ are in good agreement with the planar simulation features reported in Ref. 2. In region 4 a positive initialization relaxes toward a nonlinear stable state with oscillations close to the new equilibrium. The observed frequency of oscillation corresponds to that predicted in Ref. 9.

The critical values of $D$ defining regions with different stability properties have also been confirmed by numerical experiments for $x_{i}=100$ and $x_{i}=0.1$.

Table II presents a summary of properties for the cases studied by particle simulations. Results for each system are given separately for positive (PI) and negative initialization (NI). A list of abbreviations, used to describe the observed effects, is presented below the table. Regions 3 and 5 are always unstable for the $x_{i}$ cases tested. In regions 2,4 , and 6 there is relaxation to nonlinear equilibrium states observed to be stable, for positive initialization when $x_{i} \geqslant 1$, and also for small negative initialization when $x_{i}=0.1$.

The strongly divergent case manifests unusual dynamics after destabilization, showing a large number of accelerated particles and time-varying accelerating potentials. This case is linearly more stable (higher density threshold) than the other cases, but nonlinearly appears more fragile to disruptions (negative initialization). Conversely, the convergent case is linearly more unstable (lower density threshold). However, in numerical experiments it shows resilience to 
break up with negative initialization in even regions.

Frequency of oscillations about nonlinear equilibrium states were measured, and they turn out to be close to those predicted by linearized theory.

Numerical experiments have been carried out in the stability island of cases $x_{i}=0.1$ and $x_{i}=3.1765$ (no such stability intervals exist for $\left.x_{i}=100\right)$. The results show good agreement with the linear dispersion relation.

Studies of fine structures of the different regions and a theoretical investigation of the stability of nonlinear steady states are left for future work.

In summary, we have completed the analysis of the linear dispersion relation, and presented a survey of nonlinear behaviour with particle simulations for cylindrical Pierce diodes with radial flow. We hope the predicted properties of cylindrical diodes may interest the experimentalists in the fields of ion deposition, or microwave generation, and perhaps stimulate further theoretical studies of their nonlinear dynamics, akin to those published for planar systems.

\section{ACKNOWLEDGMENTS}

G.G. and F.T.G. are members of the Argentine National Research Council (CONICET). This work was supported by CONICET Grant No. PID-BID 594/92 on Dynamics of Plasmas and Fluids and by INFIP-CONICET (Instituto de Física del Plasma).

M.V.A. is grateful to the following Brazilian Agencies: CNPq (Conselho Nacional de Desenvolvimento Cientifico e Tecnológico), and FAPESP (Fundação de Amparo à Pesquisa do Estado de São Paulo).

${ }^{1}$ S. Kuhn, Proceedings 1987 International Conference Plasma Physics, Kiev, edited by A. G. Sitenko (World Scientific, Singapore, 1987), Vol. 2, p. 954.
${ }^{2}$ T. L. Crystal and S. Kuhn, Phys. Fluids 28, 2116 (1985).

${ }^{3}$ T. L. Crystal, P. C. Gray, W. S. Lawson, C. K. Birdsall, and S. Kuhn, Phys. Fluids B 3, 244 (1991).

${ }^{4}$ J. R. Pierce, J. Appl. Phys. 15, 721 (1944).

${ }^{5}$ J. R. Pierce, J. Nucl. Energy, Part C 2, 135 (1961).

${ }^{6}$ J. R. Cary and D. S. Lemons, J. Appl. Phys. 53, 3303 (1982).

${ }^{7}$ S. Kuhn, Phys. Fluids 27, 1821 (1984).

${ }^{8}$ B. B. Godfrey, Phys. Fluids 30, 1553 (1987).

${ }^{9}$ W. S. Lawson, Phys Fluids B 1, 1483 (1989).

${ }^{10}$ T. M. Burinskaya and A. S. Volokitin, Fiz. Plazmy 9, 453 (1983); Sov. J. Plasma Phys. 9, 261 (1983).

${ }^{11}$ M. Hörhager and S. Kuhn, Phys. Fluids B 2, 2741 (1990).

${ }^{12}$ H. Matsumoto, H. Yokoyama, and D. Summers, Phys. Plasmas 3, 177 (1996).

${ }^{13}$ G. Gnavi and F. T. Gratton, Phys. Plasmas 1, 3676 (1994).

${ }^{14}$ A. F. Alexandrov, L. S. Bogdanevich, and A. A. Rukhadze, Principles of Plasma Electrodynamics (Springer-Verlag, New York, 1984), p. 332.

${ }^{15}$ A. D. Dunn, Models of Particles and Moving Media (Academic, New York, 1971).

${ }^{16}$ A. M. Dorodnov and B. A. Petrosov, Sov. Phys. Tech. Phys. 26, 304 (1981).

${ }^{17}$ A. G. Nikonov, I. M. Roife, Y. M. Svel'ev, and V. I. Engel'ko, Sov. Phys. Tech. Phys. 32, 50 (1987).

${ }^{18}$ G. H. Miley, in Current Topics in Astrophysical and Fusion Plasmas, edited by M. Heyn and W. Kerbichler (DBV-Verlag, Graz, Austria, 1992), p. 103.

${ }^{19}$ J. W. Poukey, J. P. Quintenz, and C. L. Olson, Appl. Phys. Lett. 38, 20 (1981).

${ }^{20}$ J. W. Poukey, J. P. Quintenz, and C. L. Olson, J. Appl. Phys. 52, 3016 (1981)

${ }^{21}$ M. Abramowitz and I. A. Stegun, Handbook of Mathematical Functions (Dover, New York, 1965).

${ }^{22}$ M. V. Alves, M. A. Lieberman, V. Vahedi, and C. K. Birdsall, J. Appl. Phys. 69, 3823 (1991).

${ }^{23}$ C. K. Birdsall, IEEE Trans. Plasma Sci. 19, 65 (1991).

${ }^{24}$ PDC1 (Plasma Device Cylindrical one-dimensional), (C) 1990-1995 Regents of the University of California. Code and documentation available electronically via http://ptsg.eecs.berkeley.edu

${ }^{25}$ J. P. Verboncoeur, M. V. Alves, V. Vahedi, and C. K. Birdsall, J. Comput. Phys. 104, 321 (1993).

${ }^{26}$ C. K. Birdsall and A. B. Langdon, Plasma Physics via Computer Simulations (McGraw-Hill, New York, 1985). 